Rev. Elev. Méd. Vét, Pays Trop., 1964, 17, 3, (377-420)

\title{
Les helminthes de quelques artiodactyles sauvages appartenant aux familles des bovidés et des suidés. Ces mammifères, en République du Tchad et en R.C.A., sont-ils des réservoirs de parasites pour les animaux domestiques vivant à leur contact?
}

par GRABER (M.), DOUTRE (M.), FINELLE (P.), KERAVEC (J.), DUCROZ (G.), el MOKOTAINGAR (P.)

\section{RÉSUMÉ}

Les auteurs, au Tchad et en R. C. A., ont procédé à l'autopsie d'une cenfaine de Bovidés et Suidés squvages appartenant aux espèces suivantes : Phacochoerus aethopicus (11). Syncerus coffer dequinaxialis (5). Tragelaphus scriptus (1), Strepsiceros strepsiceros (1), Alcelophus lelwel (11), Damoliscus karrigum (7), Oryx algozel (1). Hippotragus equinus (9), Addax nasomoculatus (1), Redunco redunca nigeriensis (2), Redunco redunca (1), Adenota kob (6), Kobus defassa (8), Gazella dorcos dorcos (22), Gozella rufifrons (14), Ourebia ourebi (2), Ourebia ourebi dor$\cos (1)$, Ourebsa ourebi splendido (1).

Au total, 47 espèces d'Helminthes ont été rencontrées dont 26 spécifiques: Carmyerius exoporus, Carmyerus endopapillatus, Moniezia monardi, Avifellina sandgroundi, Crossotaenia baeri, Longistrongy/us meyeri, Longistrongylus albifrontis, Kobusinema schrenki, Hoernonehus vegliai, Parabronema skyabini, Ascaris phacochaeri, Agriostomum cursoni, Bunostomum dentatum, Daubneyı m'wanzee, Doubneyio old, Daubneyia rouboudi, Pygorginema africona, Moniezia mettami, Murshidia pugnicaudata, Artionema congolensis, Artionema scalprum, Artionema hornbyi. Artionema bicoronato, Artionema poultoni. Gozellofiloria tonganyikae, Linguatulo nuttalt.

Les 21 autres sont communes aux Artiodactyles sauvages ef aux Artiodactyles domestiques. Huit d'entre elles sont plutôt des parasiles de Buffles ou d'Antilopes qui passent, chez le zébu, le moutan et la chèvre, à la faveur des transhumances et des brassages de populations animales : Calicophoron calicophorum, Cotylophoron cotylophorum, Stephanopharynx compactus, Carmyerius spatiosus, Carmyerius papillatus. Carmyerius parvipapillatus, Stilesia hepatica et Cysticercus dromedarii.

Les treize dernières sont des espèces très courantes, à large dispersion et fortement implantées dans le pays : Paromphistomum microbothrium, Hoemonchus contortus, Sehistosoma bovis, Fasciola gigantica, Cysticercus bovis, Stilesia globipune- 
tata, Esophagostomum columbionum, Artionema lobiato-popillosa, Buckleyuris globulosa, Cooperia punctato, Avitellina woodland, Gastrodiscus aegyptıacus et Physocephalus sexolatus.

Au Tchad et en R. C. A., les Artiodactyles sauvages ne paraissent pas - pour l'instant - représenter un danger certain pour les bovins, ovins, caprins ou camelıns qui vivent à leur contact. Cependant, dans quelques cas - au demeurant limités - leur rôle ne doit pas être sous-estimé.

Les auteurs signalent, en outre, que la Faune parasitarre des Bovidés et Suldés sauvages du bassin du Chari-Logone ne diffère pas fondamentalement de celle des bassins du Nil ou du Congo et de celle des pays d'Afrique de l'Est ou d'Afrique du Sud.

L'action pathogène de ces Helmınthes el l'importance des Associations parasitaires sont également envisagées.

12 cartes de distributian géographique et 200 références bibliographiques accompagnent le présent document.

\section{INTRODUCTION}

La protection de' la faune sauvage prend, à l'heure actuelle, dans les pays d'Afrique nare, une grande importance. Les Gouvernements créent de plus en plus de réserves naturelles et les aménagent pour le tourisme et, parfois, pour la chasse.

Cette mise en valeur d'une richesse inestimable ne va pas sans présenter de nombreuses difficultés qui, en matière sanitaire, tiennent aux maladies du gibier et au rôle que peuvent jover les Artiodactyles sauvages dans la dissémination d'affections qui sont susceptibles de gagner les animaux domestiques du vorsinage.

Si les Trypanosomiases, les maladies à virus ou à bactéries, les mycoses ont falt l'objet d'études suivies (Conf. Nairobı, 1948, MC DIARMID, 1962) et sont aujourd'hur relativement bien inventoriées, il n'en esł pas de même pour les Helminthiases. En 1927, O'ROKE écrivait déjà :

«Les facteurs généralement pris en considération en matıère de protection de la faune sauvage sont l'eau, l'alimentation, le refuge ef la protection contre les ennemis. Le parasitisme, problème important mais mal compris, commence à attırer l'attention des naturalistes et des biologistes. Par sa connaissance de la biologie des parasites, le parasitologue peut rendre de grands services aux commissions de chasse et aux Instituts de faune. 》)

Depuis, bien qu'il reste beaucoup à faire (ORTLEPP, 1961), de sensibles progrès ont été réalisés en Afrique. De nombreux travaux ont vu le jour : ils ont porté :
10 Sur la description d'Helminthes nouveaux. recuelllis chez des bovidés ef des Suidés sauvages abattus sur place ou morts dans les jardins zoologiques européens, américains ou africains.

L'étude systématique des Trématodes africains est le fait d'auteurs comme LOOSS (1896), BRANDES (1898), FISCHOEDER (1901, 1902, 1903), STILES ef GOLDBERGER (1910), MAPLESTONE (1923), FUKUI (1929), STUNDKARD. (1929), TRAVASSOS (1934, 1944), NÄSMARK (1937), BEN DAWES (1946), SKRJABIN (1949), DOLLFUS (1950, 1962, 1963), PRUDHOE (1957), GRÉTILLAT (1960, 1962), DINNIK (1961).

Les Cestodes ont été décrits surtout par STILES et HASSAL (1893), 'GOUGH' (1910), THEILER (1924), BAER (1927), WOODLAND (1927), TAYLOR (1928), NAGATY (1929), SOUTHWELL (1930), FURHMANN (1932), BHALERAO (1936), JOYEUX et BAER (1936), NEVEU-LEMAIRE (1936), SPASSKY (1954-61), WARDLE et MC LEOD (1952), BAER ef FAIN (1955), MAHON (1954), YAMAGUTI (1959).

Quant aux Nématodes et aux Pentastomidés, il faut citer les ouvrages ef publications de VON. LINSTOW (1901, 1907, 1908), GEDOELST (1916), YORKE ef MAPLESTONE (1926), BAYLIS ef DAUBNEY (1926), DAUBNEY (1923, 1924 ef 1926), LE ROUX (1929), SKRJABIN et ORLOV (1934), TRAVASSOS (1934), SAMBON (1922), HEYMONS (1935), BAYLIS (1936), NEVEULEMAINE (1936), FAIN (1955), SKJABIN, SHIKHOBALOVA et SHUL'TS (1954), ALMEIDA. (1955), YEH (1959), EUZÉBY (1961, 1963), YAMAGUTI (1961), ORTLEPP (1963). 
20 Sur leur répartition géographique à l'intérieur du continent africain. Des inventaires assez précis ont été dressés.

Au Congo ex-Belge : GEDOELST $(1911,1916)$, BEAUCHAMP (1914), STUNKARD (1929), SANDGROUND (1930), STRONG et SHATTUCK (1930), WOODLAND (1935), VANDEN BERGHE (1937, 1939, 1943), RODHAIN et GILLAIN (1938), VAN DEN BERGHE et VUYLSTEKE (1936), BAYLIS (1939), DOLLFUS (1950 ef 1963), RODHAIN (1944), MAHON (1954), BAER et FAIN (1961), VUYLSTEKE (1956), PRUDHOE (1957), BAER (1959).

En Afrique du Sud et dans le Sud-Ouest africaIn : VON LINSTOW (1908), GROBBELAAR (1922), BAER (1924, 1926), DAUBNEY (1923), LANE (1923), TWAITE (1927), LE ROUX (1929 $o$ et $b, 1931,1933,1940)$, M̈ONNIG (1923, 1928, 1929, 1931, $1932 a, b$ et $c, 1933$ a ef $b$ ), MARTINAGLIA (1932, 1937), ORTLEPP (1935, 1937, 1961, 1963).

En Afrique de l'Est (Kenya, Uganda, Tanganyika, Rhodésies et Nyassaland : FISCHOEDER (1901), VON LINSTOW (1901), LEIPER (1909), INNES (1912), BOULENGER (1921), ARMFIELD (1922), SAMBON (1922), THORNTON (1924), GOODEY (1924), DAUBNEY $(1924,1926)$, WOODLAND (1928), BOULENGER (1927), TWAITE (1927), SOLOMON (1932), BAYLIS (1932, 1934, 1937), HUDSON (1934), HEYMONS (1935), SANDGROUND (1937), YEH (1955 a, 1955 b, 1958, 1959), DINNIK (1961, 1962), PESTER (1962), URQUARTH (1960).

Quelques sondages ont été réalisés toujours sur des Artıodactyles sauvages appartenant, aux familles des Bovidés et des Suidés : en Egypte par. EZZAT (1945), au Soudon par FURHMANN (1909), LEIPER (1908), BAER (1923), en Ethiopie par FURHMANN ef BAER (1943), en Angola par FISCHOEDER (1902), STILES et GOLDBERGER(1910), FURHMANN (1933), JOYEUX (1934), KREIS (1938), CAEIRO (1961) et, en Erytrée, par PELLEGRINI (1942 $a$ et $b, 1947, a, b, c$, et $d, 1950)$. COCEANI (1949), et CALL (1949).

En Afrique de l'Ouest, les travaux sont Infiniment pius rares. Ils sont dus à JOYEUX, GENDRE et BAER (1928), d̀ DOLLFUS (1929, 1932) et à MOREL (1959) pour l'A. O. F., d̀ VON LINSTOW (1899, 1904, 1907), MAPLESTONE (1923), DOLLFUS $(1929,1932)$, BAYLIS $(1936 a)$ pour le Cameroun, à RAILLIET ef HENRY (1911),
CHABAUD et ROUSSELOT (1956 a et $b, 1957$ ), GRETILLAT (1962) pour le Congo, à SANDGROUND (1930), STRONG et SHATTUCK (1933), SZIDAT (1932) pour le Liberia et la Guinée, d̀ TENDEIRO (1948 et 1951) pour la Guinée portugaise.

En ce qui concerne la République du Tchad ef la R. C. A., pays de grande chasse situés entre le Sahara et l'Equateur, la bibliographie est encore plus indigente. DOLLFUS (1950) signale trois parasites d'Hippopotamus amphibius (L.) tués entre Fort-Archambault et Fort-Lamy en 1930: Nilocotyle polycladiforme (NÄSMARK, 1937), Nilocotyle Sp. et Buxifrons maximo (NÄSMARK, 1937), et un Trématode, Colylophoron cotylophorum (FISCHOEDER, 1901), recueilli sur Damaliscus Korrigum (OGILBY) à Paoua dans le NordEst de la R. C. A.

Plus tard, CHABAUD ef ROUSSELOT (1956) décrivent sur un Adenota Kob (Erxleben) en provenance de Fort-Archambault, Setario longicauda qui a été, depuis, mis en synonymie par YEH (1959) avec Artionema pullersi (TWAITE, 1927) n. Comb.

Récemment, Cysticercus dromedor" (PELLEGRINI, 1945), a été retrouvé au Tchad sur CameIus dromedarius, Bıbos indicus, Damaliscus Korr!gum et Gazello rufifrons (GRABER, 1959).

Le présent travail a pour but de combler cette lacune et de voir si les parasites internes de Bovidés et de Suldés sauvages du Tchad et de la R. C. A. s'insèrent dans le contexte parasıtare de l'Afrique noire, tel qu'ıl vient d'être dépeint.

\section{A. ARTIODACTYLES AUTOPSIES - DATES ET LIEUX DE RÉCOLTES.}

Depuis 1954, la Section de Parasitologie du Laboratore de Farcha, grâce à l'appui précieux de nombreux vétérinaires et biologistes, a entrepris une série d'enquêtes qui ont amené au Tchad, l'autopsle d'une centaine d'animaux de chasse abattus, en grande majorité, dans les régıons centrales et méridionales du pays. En R. C. A., toujours dans les mêmes conditions, quelques sondages ont été effectués dans le centre, l'est et l'ouest du pays.

Les dates et lieux de récolte sont représentés par des numéros sur la Carte I. 
CARTE N* 1

\section{DATES ET LIEUX DE RÉCOLTES}

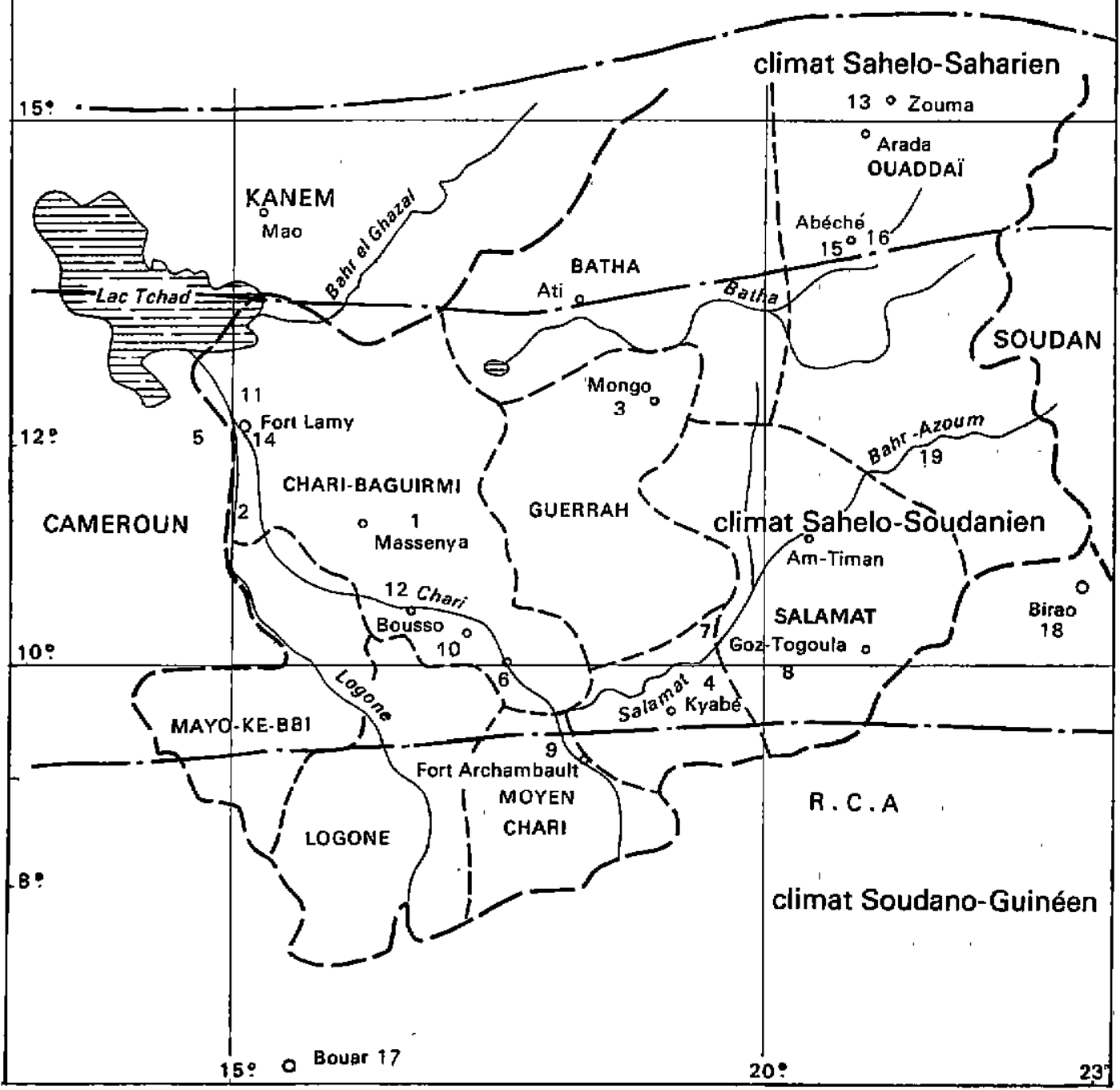

\footnotetext{
1. - Massenya, Tchad (1955, 1957, 1962)

2. - Logone birni, Cameroun (1961)

3. - Mongo, Tchad (1957)

4. - Safori Nord-Kyabé, Tchad (1961)

5. - Kousseri, Nord-Cameroun (1954, 1958)

6. - Korbol, Tchad (1963)

7. - Méré, Tchad (1962)

8. - Goz-Tagoula, Tchad (1962)

9. - Fort-Archambault, Tchad (1959)

10. - Miltou, Tchad (1963)
}

11. - Rive droite du Charı Tchad (1962)

12. - Bousso, Tchad (1954, 1955, 1963)

13. - Zouma, Tchad $(1954,1955)$

14. - Fort-Lamy, Tchad (1961, 1963)

15. - Abeche, Tchad (1963)

16. - Abougoudam, T'chad (1954)

17. - Bouar, R. C. A. (1962)

18. - Birao, R. C. A. (1958)

19. - Bahr-Azoum, Tchad (1954)

20. - Bambari, R.C.A. (1962, 1963)

21. - Ouando, R. C.A. (1963) 
TABLEAU No I

\begin{tabular}{|c|c|c|c|}
\hline & $\begin{array}{l}\text { Nombre d'animaux } \\
\text { autopstés }\end{array}$ & $\begin{array}{l}\text { Nombre d'animaux } \\
\text { gerasités }\end{array}$ & $\begin{array}{l}\text { Lieux et dates } \\
\text { de récolte }\end{array}$ \\
\hline 1) - Phacochoerug ae thiopicus (Pallas) & 11 & $1 t$ & $\begin{array}{l}1,2,3,4 \\
5,6,11,20^{+}\end{array}$ \\
\hline 2) - Syncerus caffer aequnoxialis (Blyth). & 5 & 5 & $4,18,20,21$ \\
\hline 3) - Tragelaphus scriptus (Pallas) & 1 & 1 & 17 \\
\hline 4) - Strepsiceros strepsuceros(Pallas) & $\uparrow$ & $i$ & 4 \\
\hline 5) - Alcelaphus lelwel (Heuglin) & 19 & $\$ 1$ & $4,6,7,8,9$ \\
\hline 6) - Darualiscus korrigum (Ogilby) & 7 & 7 & $1,4,8,19$ \\
\hline 7) - Oryx algazel (Okem) & 1 & 1 & 16 \\
\hline a) - Hippotragus equinus (Desmarest) & 9 & 9 & $1,4,6,6,12,19$ \\
\hline 9) - sddax nasomasulatus (Bleinville) & 1 & 1 & 16 \\
\hline 10) - Redunca redunca nigeriensig (Blaine) & 2 & 2 & 8,10 \\
\hline 11) - Redunca redunce (Pallas) & 1 & 1 & 18 \\
\hline 12) - Adenota kob (Erzleben) & 6 & 6 & $5.11,12.9$ \\
\hline 13) - Kobus defassa (Ruppel) & 8 & $\mathbf{B}$ & $1,8,7,12,14$ \\
\hline 14) - Gazella dorcas doracs (Linné) & 22 & 15 & 13. 14 \\
\hline 15) - Gazella rufifrons (Gray) & 14 & 10 & $5,13,14,15$ \\
\hline 16) - Ourebia ourebi (zimmermen) & 2 & 2 & 18,20 \\
\hline 17) - Ourebia ourebis dorcas (Schew) & 1 & 1 & 6 \\
\hline 18) - Ourebia ourebi splendida (Schw.) & 1 & 1 & 8 \\
\hline
\end{tabular}

Le tableau I donne la liste des animaux examinés : *

L'inventaire est loin d'être terminé et le nombre d'examens effectués ne permet pas encore de chiffrer avec précision l'incidence parasitaire dans chaque groupe considéré. Cependant, pour les Phacochères, les Bubales, les Waterbucks, les Hippotragues et les gazelles, les renseignements obtenus sont déjà conséquents et susceptibles d'être exploités.

\section{B. LES HELMINTHES RENCONTRES.}

II ne sera question ici que des parasites internes, les agents des Myiases faisant l'objet d'une étude à part.

\section{1. - Les helminthes du tractus digestif}

\section{Panse}

Io Poramphisfomum microbothrium (FISCHOEDER, 1910).

* Se référer aux numéros correspondants de la carle $n^{0} 1$.
Hôtes : Syncerus coffer aequinoxıalıs: I sur 5 (4, 20, 21)*

Kobus defossa : 3 sur $8(8)$

Hippotragus equinus : 2 sur $9(4,12)$

Adenota kob : 3 sur $6(5,11)$

Alcelophus lelwel : 1 sur 11 (6)

Redunca redunca nigeriensis : 1 sur $2(10)$

Damaliscus korrigum (1 sur 7) (8)

Gazella rufifrons : 1 sur 14 (5)

Paramphistomum microbothrium a été décrit pour la première fois par FISCHOEDER en 1901 et redécrit plus complètement en 1903 à partır de Paramphistomes de la panse de Gazella dorcos. Par la suite, cette espèce fut confondue avec P. cervi (ZEDER, 1790) par MAPLESTONE (1923), FUKUI (1929), STUNKARD (1929), SPREHN (1932), TRAVASSOS (1934), et DAWES (1936). NÄSMARK (1937) confirme la valıd té de l'espèce P. microbothrium sur des Trématodes de Bos taurus et de Bubolus bubalis appartenant à la collection de LOOSS ef à celle de l'expédition suédoise de 1901 au Soudan. DINNIK $(1954,1961)$ affirme

$\left.{ }^{*}\right)$ Voir carte no 1 . 
TABLEAU NO II

\begin{tabular}{|c|c|c|}
\hline Pays & Espècer animales & Auteur et date \\
\hline $\begin{array}{l}\text { Egypte } \\
\text { Egypte } \\
\text { Tanganjika } \\
\text { Congo ex-belge } \\
\text { (Parc de } 1 \text { ' Upemba) } \\
\text { Soudan } \\
\text { Congo ex-belge } \\
\text { (Sources du Congo) } \\
\text { Dganda }\end{array}$ & $\begin{array}{l}\text { Gazella dorcas } \\
\text { Gazella arabica } \\
\text { Gazella thomsoni } \\
\text { Bubalus caffer } \\
\text { Adenota variont } \\
\text { Hippotragus equinus } \\
\text { Kobus defasga crarshayi } \\
\text { Ourobia ourebi } \\
\text { Taurotragus oryx } \\
\text { Bubalus bubalis } \\
\text { Kobus defessa } \\
\text { Adenota kob } \\
\text { Redunoa bohor } \\
\text { Symcerus caffer }\end{array}$ & $\begin{array}{l}\text { Fischoeder, } 1903 \\
\text { Ezzat, } 1945 \\
\text { Yeh, } 1955 \mathrm{~b} \\
\text { Prudioe, } 1957 \\
\text { - } \\
\text { - } \\
\text { - } \\
\text { - } \\
\text { - } \\
\text { Pischoeder, } 1903 \\
\text { Sturkard, } 1929 \\
\text { - } \quad- \\
\text { - } \quad- \\
\text { Dinnik ot Coll. } 1963 \text { b. }\end{array}$ \\
\hline
\end{tabular}

que ce Paramphistome est très fréquent dans la panse des bovins et des ovins d'Egypte, du Kenya, de I'Uganda, du Tanganyika et d'Afrique du Sud, ce que confirme CAEIRO (1961) pour i'Angola.

Les spécimens des Antilopes du Tchad correspondent bien à lespèce $P$. microbothrium, tant dans leur morphologie générale, que dans la structure du pharynx, de l'acétabulum et de l'atrium génital.

Paramphistomum microbothrium a été recueilli : voir Tableau II.

Poramphistomum microbothrium est donc une espèce presque banale des Bovidés sauvages.

$2^{\circ}$ Calicophoron calicophorum '(FISCHOEDER, 1901).

Hôtes: Alcelaphus lelwel : 1 sur 11 (6)

Adenota kob: 1 sur 6 (1)

Là encore, C. colicophorum a longtemps été mis en synonymie avec Gigantocotyle explanatum (CREPLIN, 1847) par MAPLESTONE (1923), FUKUI (1929), STUNKARD (1929), SPREHN (1932) rétablił l'espèce de FISCHOEDER dans sa valıdité.

Ce Trématode est également un parasite des ruminants domestiques d'Afrique noire (MÖNNIG, 1928, GRETILLAT, 1960, CAEIRO,
1961) : il est cependant beaucoup plus rare que le précédent.

$3^{\circ}$ Cotylophoron colylophorum (FISCHOEDER, 1901).

Hôtes : Alcelaphus lelwel : 3 sur $11(4,6,7)$, * Syncerus caffer : 3 sur $5(4,20,21)$

STILES et GOLDBERGER créent le genre en 1910 avec deux espèces : C. cotylophorum ef C. indicum que MAPLESTONE (1923) rassemble en une seule. Cette erreur subsiste jusqu'à NÄSMARK (1937) qui, après avoir validé les deux espèces précédentes, en ajoute deux autres : C. füllebormi et C. jacksoni. En 1953, PRICE et MC INTOSH révisent le genre Cotylophoron et finalement n'admettent plus que cinq espèces :

C. Cotylophorum (FISCHOEDER, 1901) : Afrique et Asie

C. Panomensis (PRICE et MC INTOSH, 1953) Apérıque

C. jacksoni (NÄSMARK, 1937) : Afrique

C. fülleborn! (NÄSMARK, 1937) : Afrique

C. noveboracensis (PRICE et MC INTOSH, 1953): Amérique

C. ovatum (HARSHEY, 1934), C. orientalis

(*) Voir carte no 1 . 
(HARSHEY, 1934) C. elongatum (HaRSHEY, 1934) et $C$. okopi (= Congolense, BAER 1936) LEIPER, 1935, passent dans le genre Orthocoelium, STILES et GOLDBERGER, 1910, avec une nouvelle sous-famille, celle des Orthocoelimae. En outre, PRICE et Mc INTOSH (1953), à cause de lo structure musculaire de l'acetabulum, éliminent $C$. indicum et le transfèrent dans le genre Amphistomum, sous le nom d'A. thopari.

Les caractères des Cotylophoron recueillis au Tchad et en R. C. A., en particulier la présence d'un bulbe oesophagien, indiquent bien qu'il s'agit de l'espèce Cotylophoron cotylophorum.

Sur un certain nombre d'exemplaires, les testicules ne sont pas disposés rigoureusement en diagonale, comme le prétend NÄSMARK (1937, p. 500). Le même phénomène a été observé également por STUNKARD (1929), puis par DOLLFUS (1963).

Cotylophoron cotylophorum a été retrouvé :

Par STUNKARD (1929) chez Neotragus pygmaeus et Adenota Kob (ex-Congo Belge).

Por JOYEUX, GENDRE ef BAER (1928) chez Alcelaphus maior (Dahomey), par ORTLEPP (1935) chez Boselophus tragelocomelus (Afrique du Sud).

Ces auteurs se sont basés sur le traval de MAPLESTONE (1923): il est donc possible que certans exemplaires considérés comme des C. cotylophorum ne le soient pas en réalité.

Plus tard, DOLLFUS (1950) signale ce Trématode chez Domoliscus Korrigum en R. C. A. et, lors de l'exploration des pares nationaux de l'ex-Congo Belge, VAN DER BERGHE (1934) et PRUDHOE (1957) le mettent en évidence chez Bubalus caffer. Tragelophus scriptus, Adenota vardoni, Kobus defossa et Taurotragus oryx.

\section{$4^{\circ}$ Stephanophorynx compactus (FISCHOEDER,} 1901)

Hôtes: Kobus defossa: 1 sur $8(8)+$

Hippotrogus equinus : 1 sur 9 (4)

Redunca redunco nigeriensis : 1 sur 2 (10)

Adenoto kob : 1 sur 6 (5)

II existe actuellement trois espèces de Stephonopharynx :

a) Stephonopharynx compoctus décrit par FISCHOEDER en 1901 à partir d'exemplaıres de la panse de Bos taurus (Afrique) et redécrit par MAPLESTONE (1923) d'après des parasites immatures récoltés dans l'estomac de trois waterbucks (Cobus) tués à N'Goa en Rhodésie. Les spécimens de MAPLESTONE ont été réexaminés par NÄSMARK en 1937.

GRETILLAT (1960a et $b$ ) donne d'utıles renseignements sur la structure du diverticule pharyngien de Stephonopharynx compactus ; les Trématodes provenaient de l'appareil digestif de zébus sacrifiés à l'abattorr de Fort-Lamy-Tchad.'

b) Stephonopharynx secundus (STUNKARD, 1929) de l'estomac de Redunca bohor (= Redunco (redunco) redunca) au Congo ex-Belge.

c) Stephonopharynx coilos (DOLLFUS 1963) de l'estomac d'Hippotragus equinus à Kında (Congo ex-Belge).

Une série de coupes (une quinzaine au total) a été pratiquée sur les exemplaires tchadiens: les caractères anatomiques correspondent exactement à ceux de $S$. compoctus, tel's quils ont été représentés par NÄSMARK (1937, p. 391, fig 62-3; p. 420, fig. B3, pl. XIII, fig. 4-7), par MAPLESTONE (1923, p. 169, fig. 12) et por FISCHOEDER (1901).

Par rapport à S. collos (DOLLFUS, 1963), la poche retrobuccale de $S$. compactus n'a pas la même amplitude ef il manque, dans cette même poche, le revêtemenł papillaire si caractéristique de S. collos.

$5^{\circ}$ Carmyerius spatiosus (BRANDES, 1898).

Hôtes : Syncerus caffer aequinoxialis : 1 sur 5 (4) *

Hippotrogus equinus : 1 sur 9 (12)

Adenota kob : 1 sur 6 (5)

Redunco redunca nigeriensis : 1 sur 2 (10)

Damaliscus korrigum : 1 sur $6(8)$

Alcelophus lelwel : 2 sur 11 (6)

Kobus defassa : 2 sur $8(7,8)$

60 Carmyerius papillatus (GRETILLAT, 1962).

Hôtes : Kobus defassa: 1 sur 8 (7)

Adenota kob : 1 sur 6 (11)

70. Carmyerius exoporus (MAPLESTONE, 1923)

Hôte : Damoliscus korrigum : 1 sur 7 ( 8 )

$8^{\circ}$ Cormyerius parvipapillatus (GRETILLAT, 1962).

Hôtes : Kobus defassa : 1 sur 8 (8)

Domoliscus korrigum : 1 sur 7 (8)

Adenota kob : 1 sur 6 (11)

(*) Voir carle $n^{0} 1$ 
90 Carmyerius endopapillatus (DOLLFUS, 1962). Hôte: Syncerus coffer aequinoxialis : 1 sur 5 (4).

L'histoire des Carmyerius, comme celle de Paromphistomum microbothrium est fort complexe. Ce sont des amphistomata appartenant d̀ la famille des Gastrothylacidae.

Cinq espèces décrites par FISCHOEDER (1901, 1902,1903) ont été séparées pour former le genre Cormyerius (STILES et GOLDBERGER 1910). Par la suite, le genre s'est enrichi de trois espèces nouvelles: C. bubalis (INNES, 1912), C. wenyoni (LEIPER, 1908) et C. cruciformis (LEIPER, 1910).

MAPLESTONE (1923), puis FUKU! (1929) réduisent le nombre des espèces à cinq, puis à quatre : C. gregarius, $C$. spatiosus, $C$. wenyon et $C$. exoporus et considèrent comme synonymes de $C$. spatiosus, $C$. synethes, $C$. minutus, $C$. mancupotus, $C$. bubalss, $C$. wellmani ef $C$. cruciformis. Cette classification est suivie par TRAVASSOS 1934 et NEVEU-LEMAIRE (1936).

DAWES (1936) supprime même le genre Carmyerius et ne lassse subsister que le genre Gastrothylax (POIRIER, 1883).

Cette opinion n'est pas partagée par tous les auteurs. SKRJABIN (1949) ef YAMAGUTI (1958) conservent les trois genres Gostrothylax, Carmyerius et Fischoederius.

GOLVAN, CHABAUD et GRETILLAT (1957) démontrent la validité de chacune des espèces du genre Cormyerius dont GRETILLAT (1960) donne une clef de détermination pour 11 d'entre elles.

En 1962, DOLLFUS, puis GRETILLAT font la description de trols Carmyerius nouveaux d'Afrique centrale : $C$. endopopillatus de Syncerus caffer au Congo ex-Belge, Carmyerius papillatus et $C$. parvipapillatus de Kobus defassa à Brazzaville.

Dans un récent travail, DOLLFUS (1963) reprend la question, en insistant surtout sur un certain nombre de détails - forme de la poche ventrale, longueur des caecums intestinaux permettant la différentıation des 16 espèces de Carmyerius actuellement connues.

Parmi les Carmyerius rencontrés au Tchad chez les Artiodactyles sauvages, trois d'entre eux méritent de retenir plus particulièrement l'attention. Ce sont des Gastrothylacidae dont le pore génital est couvert de papilles :

- Chez C. endopapillatus, elles débordent largement les parois antérieures de la poche ventrale pour rejoindre, dans certaıns cas, le revêtement papillaire de la région antérieure du corps.

- Chez $C$. popillotus, Il existe un très vaste atrium génital ( $500 \mu$ environ) entouré de fibres musculaires et au fond duquel se trouve le pore génital. Sa paroi est tapissée de papilles de 20 à $30 \mu$. La cavité génitale débouche à peu près au même niveau que l'orifice de la poctie ventrale. l'œsophage mesure'600 à $700 \mu$ de long.

- Chez C. porvipapillatus, on a affaire à un puissant sphincter qui s'ouvre dans la poche ventrale à $600 \mu$ de son orifice : c'est là un caractère à peu près constant. Le sphincter génıtal est parsemé, en surface, de petites papilles dont la taille ne dépasse pas $12 \mu$. L'œsophage est court $(450 \mu)$.

Pour les espèces plus anciennes, il convient de noter que Carmyerius spatiosus a été découvert chez Hippotragus equinus en Rhodésie (MAPLESTONE, 1923) et chez Hippotragus beckeri au Soudan (BAER, 1923), Carmyerius exoporus chez Tragelaphus spekei au Nyassaland (MAPLESTONE, 1923 ) et Bubalus coffer au Congo ex-BelgePrudhoe, 1957).

\section{Caillette.}

10 Longistrongylus meyeri (LE ROUX, 1931). Hôtes : Hippotragus equinus : 1 sur $9(12)+$ Alcelaphus lelwel : 1 sur $11(7)$

Kobus defassa: 1 sur $8(8)$

Autres hôtes :

Alcelaphus buselaphus (Afrique du Sud, LE ROUX 1931)

1955 b)

Gazella thomsont (Tanganyika, YEH, 1963 b)

Syncerus caffer (Ugandá, DINNIK et Coll.

$2^{\circ}$ Longistrongylus albifrontis (MÖNNIG, 1931. Hôtes: Alcelaphus celwel: 1 sur 11 (8)

Autres hôtes :

Damaliscus abifrons (Afrique du Sud, MÖNNIG, 1931)

Antidorcos morsupialis (Afrique du Sud, MÖNNIG 1933 a)

(*) Voir corte no 1. 
30 Kobusinema schrenki (ORTLEPP, 1939) ORTLEPP, 1963, nov. Comb.

Hôtes: Hippotragus equinus : 1 sur 9 (12)

Autres hôtes :

Cobus ellipsiprymnus (Afrique du Sud, ORTLEPP, 1939)

Adenota kob thomosi (Uganda, DINNIK, 1963 a)

Ces Trichostrongylidae sont essentiellement africains. La première description remonte à LE ROUX (juin, 1931) pour Longistrongylus meyeri parasite d'Alcephalus buselophus, suivie de celle de Bilgakeo albifrontis (MÖNNIG, août 1931) pour un Nématode de Damaliscus albifrons. Au genre Bigalkea, MÖNNIG (1932 c, 1933 o) rattache B. sabie, et, en 1939, ORTLEPP décrit Longisirongylus schrenki du Waterbuck, Cobus ellipsiprymnus.

.TRAVASSOS (1937) dons sa révision des Trichostrongy/idae ne reconnaît pas la validité du genre Bigalkea qu'il met en synonymie avec Longistrongylus, de sorte que ce genre comprend, en 1939. quatre espèces :

L. meyeri (LE ROUX, 1931)

L. albifrontis (MÖNNIG, 1931)

L. sabie (MÖNNIG, 1932)

L. Schrenki (ORTLEPP, 1939)
En 1954, SKRJABIN, SHIKHOBALOVA ef SCHULTS acceptent le transfert de B. albifrontis dans le genre Longistrongylus, mais conservent le genre Bigalkea pour B. sabie et B. schrenkı. Ces auteurs estiment que la distinction entre les deux genres est parfaitement valable, car le genre Longistrongylus est caractérisé par une côte dorsale double, tandis qu'elle est simple dans le genre Bigolkeo.

ORTLEPP (1963) n'est pas de cet avis : il considère en effet que, puisque l'espèce type du genre Bigolkea, B. albifrontis, a été placée dans le genre Longistrongylus, le genre Bigalkea perd so raison d'être et, conformément aux règles internationales de nomenclature zoologique, devient synonyme de Longistrongylus. II propose en remplacement la création du genre Bigalkinema ef, pour L. schrenkı, celle du genre Kobusinemo.

En définitive, toujours d'après ORTLEPP (1963), Il existerait pour l'ensemble de ces Trichostrongylidae trois genres :

a) Le genre Longistrongylus (LE ROUX, 1931, SKRJABIN ET SHIKHOBALOVA, 1954).

b) Le genre Bigalkinema (ORTLEPP, 1963).

c) Le genre Kobusinemo (ORTLEPP, 1963).

Leur différenciation se fait d'après l'aspect de la bourse caudale du mâle. Le Tableau no III résume les principaux caractères distinctifs :

TABLEAU NO III

\begin{tabular}{|c|c|c|c|}
\hline & Longistrongylus & Bigalkinema & Kobusinema \\
\hline 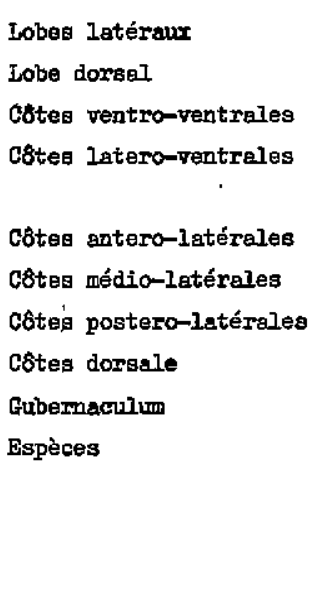 & 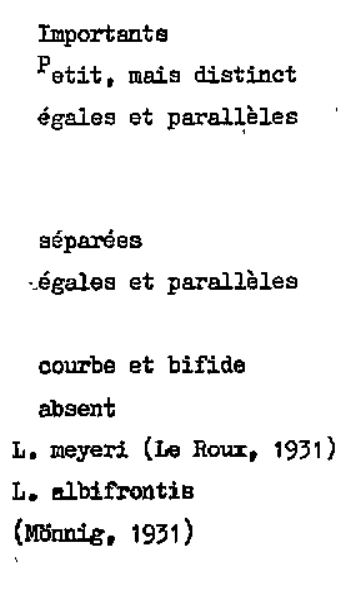 & $\begin{array}{l}\text { non distinct } \\
\text { divergentes } \\
\text { simple } \\
\text { présent } \\
\text { B. sabie (Mtinnz.8. 1932) } \\
\text { Hov. Comb. } \\
\text { B. nemaquensis } \\
\text { Oxtlepp, 1963 }\end{array}$ & $\begin{array}{l}\text { distinct } \\
\text { très inportmtes } \\
\text { plus grosses que les } \\
\text { autres cótes } \\
\text { égales et parallèles } \\
\text { aimple } \\
\text { absent } \\
\text { K.schrenla Ortlepp, } 1939 \\
\text { Nov. Camb. }\end{array}$ \\
\hline
\end{tabular}


40 Haemonchus contorfus (RUDOLPHI, 1803, COBBOLD, 1898).

Hôtes: Alcelaphus lelwel : 1 sur 11 (8)

Gazella dorcos : 10 sur $22(13,14)$
Gazella rufifrons : 1 sur 14 (13)

Haemonchus contortus est très largement répandu chez les Antilopes d'Afrique noire. (Tab. IV)

PABLFAU NO IV

\begin{tabular}{|c|c|c|c|}
\hline Points de récolte & Éspèces anumales & Auteurs & Date \\
\hline $\begin{array}{l}\text { Londres - Zoo } \\
\text { Autriche - Zoo } \\
\text { Sénégambie - Nigéria } \\
\text { Soudan } \\
\text { Tanganyika } \\
\text { Sénégal } \\
\text { Uganda } \\
\text { Congo ex-belge } \\
\text { Zoo Prétoria (Afr.du sud) } \\
\text { Etat d'Orange (Afr.du sud) ' } \\
\text { Kenya }\end{array}$ & $\begin{array}{l}\text { Hippotragus equinus } \\
\text { Gazella rufifrona } \\
\text { Gazella rufifrong } \\
\text { Gazella thomsoni } \\
\text { Gazella rufifrons } \\
\text { Hippotragus equinus } \\
\text { Adenota kob thomasz } \\
\text { Limotragus speker } \\
\text { Damaliscus albifrons } \\
\text { Tragelaphus sylvaticus } \\
\text { Antidorcas marsupialis } \\
\text { Raphiceros campeatris } \\
\text { Cobus ellipgiprymnus } \\
\text { Danaliscus albifrons } \\
\text { - }\end{array}$ & $\begin{array}{l}\text { Vevers } \\
\text { Gebauer } \\
\text { Ezaat } \\
\text { Yeh ' } \\
\text { Morel } \\
\text { Dinnik } \\
\text { Van den Berghe } \\
\text { Monnig } \\
\text { - } \\
\text { - } \\
\text { - } \\
\text { - } \\
\text { Monnig } \\
\text { Martinaglia } \\
\text { Monnig } \\
\text { - } \\
\text { - }\end{array}$ & $\begin{array}{l}1922 \\
1932 \\
1945 \\
1956 \text { b } \\
1959 \\
1963 \\
1943 \\
1931 \\
- \\
- \\
- \\
- \\
1932 \text { aे } \\
1937 \\
1933 \text { à } \\
- \\
-\end{array}$ \\
\hline
\end{tabular}

\section{Haemonchus sp.}

Hôtes : Kobus defosso : 1 sur 8 (8)

Syncerus caffer aequinoxialis: 1 sur 3 (4)

La détermination de ces Trichostrongles n'a pas été possible : les exemplaires étaient en mauvais état ef aucun mâle n'était visible dans le lot.

60 Haemonchus vegliai (LE ROUX, 1929). Hôte : Strepsiceros strepisiceros : 1 sur 1 (4)

Cette espèce, dépeinte pour la première fois par LE ROUX (1929 a) chez le même hôte, s'apprécie en mesurant la distance qui sépare l'extrémité de chaque spicule du crochet qu'it porte, distance qui est de $40-50 \mu$ pour le spicule droit et de 28-50 $\mu$ pour le spicule gauche.

70 Parabronema skrjabini (RASOVSKA, 1924).
Hôte : Hippotragus equinus : 1 sur 7 (12).

Le parasite rencontré chez l'Hippotrague semble très voisin de Parabronema skrjabını et nous la laissons provisoirement sous ce nom, en attendant d'autres exemplaires. L'espèce a été décrite d'abord au Turkestan. Elle a été revue depuis chez la girafe (Afrıque du Sud), I'Ibex (Zoo du Caıre), l'Okapi (Rép. démocratıque du Congo) et en Uganda chez Syncerus Coffer (DINNIK ef Coll., 1963 b).

\section{Estomac.}

Physocephalus sexalatus (MOLIN, 1860)

Hôte : Phacochoerus aethropicus : 1 sur 11 (6)

Intestin grêle. 


\section{CESTODES}

10 Moniezia monordi (FURHMANN, 1931).

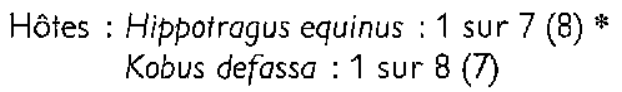

Hôtes : Hippotragus equinus : 1 sur $7(8)$ * Kobus defassa: 1 sur $8(7)$

Moniezıa monardi, brièvement identifié par FURHMANN en 1931, a été complètement redécrit par le même auteur en 1933 à partir d'un Cestode de Redunca amadirum récolté à Rio M'bali en Angola et par DINNIK (1963 a) chez Adenota kob thomasi en Uganda.

La taille du parasite, sa largeur (12 mm), les dimensions du scolex (1,7-1,8 mm) correspondent ¿̀ celles données par DINNIK. D'autre part, l'aspect des testicules qui vont bien au-dessus de l'ovaire jusqu'au niveau des canaux excréteurs,' l'anatomie du vagin entouré d'une épaisse couche de cellules glandulaires et sa termınaison dans l'atrium génital au moyen d'un sphincter puissant, la forme en boutelle de la poche du cirre et sa longueur, la difficulté de mettre en évidence les glandes interproglotidiennes en « rosette», tous ces éléments incitent à penser que l'on a bien affaire chez l'Hippotrague et le Waterbuck du Tchad à Moniezı monardi.

Le genre Moniezıa a subi de multiples vicissitudes et le nombre d'espèces admises a varié sensiblement selon les auteurs (STILES et HASSALL, 1893 ; THEILER, 1924 ; BAER, 1927, TAYLOR, 1928). WARDLE ef MCLEOD (1952) reconnaissent cinq espèces valables dont $M$. monard qui s'apparente étroitement à Moniezıo expanso.

II est bon de rappeler que $M$. exponsa ef $M$. benedeni sont des Cestodes particulièrement fréquents chez les grandes Antilopes d'Afrique tropicale (BAER, 1923, 1926, 1927), MAHON (1954), BAER ef FAIN (1955).

20 Moniezia mettami (BAYLIS, 1934).

Hôte : Phacochoerus aethiopıcus : 2 sur 11 (4).

Ce parasite a été découvert par BAYLIS (1934) en Uganda et revu par MAHON (1954) םu Congo ex-Belge.

$3^{\circ}$ Avitellina sandgroundi (WOODLAND, 1935).

Hôtes: Alcelaphus lelwel : 1 sur 11 (8)

Domaliscus korrigum : 1 sur 7 (4)

Adenota kob: 1 sur 6 (5).

Hippotragus equinus : 2 sur $7(4,6)$

(*) Voir carte no 1.
WOODLAND (1928) crée, pour les Avitellina d'Antilopes, le genre Anootypus avec A. edifontoneus de Taurotragus oryx et $A$. ricord du Waterbuck. FURHMANN (1931) rajoute $A$. monardi de Taurotragus oryx. SOUTHWELL (1929) pense que les caractères généraux du genre Anootypus ef du genre Avitellina ne permettent pas de les séparer, ce que confirme SPASSKY (1951) pour qui Anootypus et Ascotoenia sont synonymes d'Avitellina. Actuellement, les auteurs ne sont pas tous d'accord sur ce point (SINGH et PANDE, 1963).

Avitellina sandground des Antilopes du Tchad est caractérisé par l'extrême brièveté des segments, un scolex très large (plus de $2 \mathrm{~mm}$ ) des testıcules disposés sur quatre colonnes, une poche de cirre de $121 \mu$ et une vulve de $220 \mu$.

La distribution géographıque d'Avitellına centripunctato en Afrique est très large: Afrique du Sud (BAER, 1926 ; MÖNNIG, 1928), Congo ex-Belge (MAHON, 1954 ; BAER et FAIN, 1955) Guinée portugaise (TENDEIRO, 1951), Ethiopie (FURHMANN et BAER, 1943), Soudan (BAER, 1923). Les hôtes cités sont nombreux : Cephalophus nigrifons, Damalıscus korrigum. Tragelaphus scriptus, Syncerus caffer, Cephalophus maxwelli, Cepholophus rufilatus, Hippotragus niger, Bubalus caffer, Taurotragus oryx, Sylvicapra grimmia, Oryx beisa, Gazella granti, Oreotragus oreotragus, Pediotragus horstocki, Hippotragus equinus.

La question se pose, ainsi que SANDGROUND (1935) le faisait déjà remarquer, de savoir s'il faut, dans tous les cas, incriminer Avitellina centripunctato. MÖNNIG (1928, p. 804) écrit, en effet, à propos de ce Cestode: "This is a narrow tapeworm, usually not over $3,5 \mathrm{~mm}$ broad, with the uterus in ripe segments showing as an opaque line running down the middle line: the segmentation is very fine $)$, description qui, pourrait en imposer pour Avitellina sondground.

40 Avitellino woodland! (BHALERAO, 1936). *

Hôtes: Addax nasomaculatus : 1 sur 1 (16). Oryx algazel : 1 sur 1 (16).

La descriptıon originale de BHALERAO (1936) a été faite à partir d'un Cestode recueilli dans l'intestin d'une chèvre à Muktesar (Indes). MALEK (1959) assımile à cette espèce des Anoplocephalidés de dromadaires abattus à Kartoum, Kosti

* que SPASSKY (1951) considère comme une forme d'A. centripunciata. 
et El Fascher. Cette dernière localité est elle-même approvisionnée par l'Ouaddaī, région NordEst du Tchad où Avitellina woodlandi semble assez fréquent chez les ovıns et les camelins. Le contact étroił entre chameaux et ruminants sauvages qui utılisent les mêmes pâturages et les mêmes puits dans les fonds d'Ouadis explique la présence pour le moins paradoxale - de ce Cestode chez les Antilopes des zones pré-désertiques.

Le diagnostic de l'espèce ne soulève pas de difficultés : la longueur de la poche du cirre par rapport à celle de la vulve, les dimensions du scolex et l'aspect des organes parutérins lèvent le doute.

\section{$5^{0}$ Stilesia globipunctata (RIVOLTA, 1874).}

Hôte : Hippotragus equinus : 1 sur $9(6) *$

Le «Ténia frisé » a été signalé dans le duodénum de diverses Antilopes d'Afrique centrale et méridionale, notamment Cobus ellipsiprymnus en Afrique du Sud (BAER, 1927), Gazello granti locuum en Ethıopie (FURHMANN et BAER, 1943), Cepholophus nigrifons au Congo ex-Belge (MAHON, 1954), et Ourebia ourebiau Kenya(ROUND, 1962).

\section{NEMATODES}

10 Agriostomum cursoni (MÖNNIG, 1932).

Hôtes : Kobus defossa : 1 sur $8(8) 15$ exemplaires. Damaliscus korrigum: 1 sur 7 (8) 41 exemplaires

Alcelaphus leiwel : 1 sur 11 (8) 70 exemplaires.

Le bord antérieur de la capsule buccale est armé de fortes dents disposées par deux. La dent interne de chaque parre est plus courte que la dent externe et les deux lancettes du fond de la capsule sont assez peu proéminentes. Les mâles mesurent de 10 à $12 \mathrm{~mm}$ et les femelles de 15,8 à $16 \mathrm{~mm}$. Les spicules des mâles varient de 1,15 à $1,48 \mathrm{~mm}$.

Ces caractères ne sont ceux ni d'Agriostomum gorgonis de Gorgon taurinus (LE ROUX, 1929 b), ni d'Agriostomum vryburgi de Bos indicus (RAILLIET, 1902, LANE, 1923 ; WARE, 1925 ; SMIT et NOTOSOEDIRO, 1923), ni d'Agriostomum equidentatum d'Antidorcas marsupialis (MÖNNIG, 1929). Les Agriostomes des Antilopes du Tchad semblent donc pouvoir être rapportés d̀ l'espèce $A$. cursoni.

La localisation de ces Ancylostomidés est curieuse : A. vryburgi, duodénum ; A. equidentotum, côlon et duodénum; A. gorgonis, iléon. Quant à A. cursoni, MÖNNIG dit l'avorr recueilli dans l'intestin grêle.

Nos exemplaires ont été prélevés dans le duodénum (mélangés à Bunostomum dentatum) chez Kobus defassa, dans le caecum pour Domaliscus korrigum et Alcelaphus lelwel (en même temps que des Pygarginema).

Autres hôtes: Strepsiceros strepsiceros ef Alcelaphus coama - Parc national Kruger - Afrique du Sud (MÖNNIG, 1933 a), Damaliscus lunatus Bechuanaland (MÖNNIG, 1932, o).

$2^{\circ}$ Bunostomun dentatum (MÖNNIG, 1931).

Hôtes : Kobus defasso : 2 sur 8 (8). Hippotragus equinus : 1 sur 9 ( 8 ) 23 exemplaires.

La même année (1931), MÖNNIG et MAPLESTONE décrivent, le premier, Bunostomum dentatum chez un Kobus defossa mort au jardin zoologique de Prétoria et le second. Bunostomum cobi, chez un Cobus ellipsiprymnus du jardin zoologique de Calcutta. BAYLIS (1936 b) pense que les différences tiennent surtout dans les mensurations ef que $B$. dentotum doit être mis en synonymie avec B. cobi. RAMANUJACHARI et ALWAR (1951) ne sont pas tout à fait du même avis, car $B$, dentatum possède un gubernaculum de $135 \mu$, tandis que $B$. cobi n'en a pas.

Les exemplaires tchadiens montrent une capsule buccale tantôt de type dentatum (MÖNNIG, 1931, p. 242, fig. 14 et 15), tantôt de type cobi (MAPLESTONE, 1931, p. 165, fig. 148). D'autre part, la longueur est de $12 \mathrm{~mm}$ pour le mâle et de $16 \mathrm{~mm}$ pour des femelles immatures. II existe un gubernoculum ef les spicules étroits et ailés mesurent $637 \mu$.

L'ensemble des caractères semble rapprocher les Bunos-tomes de Kobus defassa et d'Hippotragus equinus de Bunostomum dentatum. Ce nom sera donc conservé en attendant de plus amples renseignements.

Tout récemment, DINNIK (1963 a) a retrouvé le même parasite chez Adenota kob Thomosı en Uganda.

$3^{\circ}$ Ascaris phacochori (GEDOELST, 1916). Hôte: Phocochoerus aethiopicus : 2 sur 11 (4) *

$\left(^{*}\right)$ Voir carte $n^{0} 1$. 
40 Coopria punctafa (VON LINSTOW, 1906, - RANSOM, 1907).

Hôte : Kobus defosso: 1 sur $8(7)$ *

Autre hôte : Damaliscus albıfrons en Afrique du Sud (MÖNNIG, 1932 a).

\section{Cooperia Sp.}

Hôte : Gazella rufifrons : 2 sur 14 (15).

L'absence de mâles et le mauvaıs état du matériel n'ont pas permis de déterminer avec précision l'espèce en cause.

\section{1. - Gros intestin-côlon}

10 Gastrodiscus aegyptiacus (COBBOLD, 1876).

Hôte : Phacochoerus aethiopicus : 4 sur 11 (1, $2,3,4 ; 6,20) *$.

Trématode très fréquent dans toute la vallée du Chari.

Normalement, Gastrodiscus aegyptiacus est un parasite de chevaux, d'ânes, de zèbres et de mulets. II fut découvert en 1870 à Zagazig (Egypte) et revu, depuis, en plusieurs points d'Afrique, des Indes, et des Antilles.

Chez le phacochère, le Trématode a été rencontré à N'Goo en Rhodésie par MAPLESTONE (1923), à Goungoun au Dahomey par JOYEUX, GENDRE ef BAER (1928), à Niafouké dans la vallée du Niger par DOLLFUS (1932) et au Congo ex-Belge par STUNKARD (1929) et PRUDHOE (1957).

$2^{\circ}$ Daubneyia m'wanzee (DAUBNEY, 1924).

Hôte : Phacochoerus aethiopicus: 4 sur 11 $(3,4,5)$ *

30 Daubneyia oldi (GOODEY, 1924).

Hôte : Phacochoerus aethiopicus : 2 sur $11(1,6)$ *

40 Doubneyia rouboudi (DAUBNEY, 1926).

Hôte: Phoecochoerus aethiopicus : 3 sur 11 $(1,3,6)$ *

LE ROUX (1940) à partir d'exemplaires récoltés au Ghana sur des Phacochères, pense qu'il ne s'agit pas là de vrais Oesophagostomes ef qu'ils ne doivent pas être inclus dans le genre Oesaphagostomum MOLIN 1861. II propose la création du genre Daubneysa qu'il décrit ainsi : « extrémité antérieure avec ou sans renflement cuticulaire. Coronule externe composée de

(*) Voir carte no 1.
6 à 8 éléments. Collıer buccal profondément déprımé dorsalement et ventralement, entraînant la formatıon de deux «lèvres » latérales. Capsule buccale à parois épaisses. Papilles céphaliques subdorsales et subventrales longues. Papilles cervicales longues et minces. Extrérnité postérieure de la femelle courte ef pointue, plus ou moins courbée dorsalement $\gg$.

Ces caractères apparentent les Daubneyia aux genres Trichonema (COBBOLD, 1874) et Murshidra (LANE, 1914).

Sept espèces font partie du genre Daubneyia. Elles ont été bien étudiées par DAUBNEY (1924. 1926), DOODEY (1924), THORNTON (1924), NEVEU-LEMAIRE (1927) et SANDGROUND (1937). Les exemplaires venaient d'Afrique de l'Est et de Somalie.

Les Oesophagostomes des Phacochères sont souvent associés entre eux.

5o Oesophagostomun (Proteracrum) columbianum (CURTICE, 1890), RAILLIET ef HENRY, 1913.

Hôte: Gazella rufifrons: 2 sur $14(15,5)$ *

$6^{\circ}$ Murshidia pugnicaudata (LEIPER, 1909).

Hôte : Phacochoerus aethropicus : 1 sur 11 (5).

7o Pygarginema ofricana (CHABAUD et ROUSSELOT, 1956).

Hôte: Alcelophus lelwel : 1 sur 11 (8).

Deux mâles-de Pygarginema ont été isolés d'un lot de 70 Agricostomum cursoni.

L'aspect de la tête, l'ornementation de la queve, la longueur des spicules rapprochent le spiruridé d'Alcelaphus lelwel de Pygarginema africana tel qu'il a été figuré par CHABAUD et ROUSSELOT (1956). L'absence de femelles ne permet pas de conclure définitivement.

Par ailleurs, le pornt d'implantation est différent caecum, au lieu du duodénum. Ce qui a été dit plus haut, à propos d'Agriostomum cursoni auquel ce paraste était mêlé, pourrait également, sous réserve de vérifications ultérieures, s'appliquer à Pygarginema ofricana.

Autre hôte: Cepholophus dorsalis castoneusCongo Brazzaville.

$8^{\circ}$ Buckleyuris globulosa (VON LINSTOW, 1901).

Hôte : Kobus defassa : 1 sur $8(7)^{*}$ Gazella dorcas : 1 sur 22 (14)

(*) Voir carte no 1. 
Gazello rufifrons : 1 sur 14 (14)

Hippotrogus equinus: 4 sur $9(6,8,12)$.

Les caractères de ceffe espèce ont été fixés par SPREHN (1927), BAYLIS (1932), GEBAUER (1932), ORTLEPP (1937) et surtout SARWAR (1959) qui remodèle le genre Trichuris en le scindant en trois (genres Buckleyuris, Rudolphio ef Salamaro).

Buckleyuris globulosa infeste Gazella albonotata, Gazella Dama, Gazella dorcas, Gazella leptoceros et Gazella rufifrons en Egypte (EZZAT, 1945), Antidorcas morsupialis et diverses gazelles en Afrique du Sud (ORTLEPP, 1937), Adenota kob Thomasi en Uganda (DINNIK, 1963 a) et Gazelio rufifrons au Sénégal (MOREL, 1959).

Buckleyuris globulosa est, 'chez les ruminants domestiques du Tchad, bien plus abondant que Buckleyuris ovis : la proportion est d'environ 9 pour 2.

\section{II. - Les helminthes de la cavité périfonéale et des plèvres.}

10 Artionema congolensis (RAILLIET et HENRY, 1911).

Hôfe: Phocochoerus aethiopicus : 2 sur $11(1,3)$.

C'est un Filariidé banal de Phacochoerus oethiopicus et de Potamocherus porcus au Congo (RAILLIET et HENRY, 1911), au Congo ex-Belge (GEDOELST, 1916 ; VAN DEN BERGHE ef VUYLSTEKE, 1936 ; BAYLIS, 1939 ; VUYLSTEKE, 1956) ef en Afrique portugaise de l'Esł (MÖNNIG, 1928).

$2^{\circ}$ Artionema scalprum (VON LINSTOW, 1908) N. Comb.

Hôtes: Gozella dorcas : 15 sur 22 (15). Gazella rufifrons : 6 sur $14(13,15)$

Ourebia ourebi : 2 sur $2(18,20)$.

La premıère description remonte à VON LINSTOW. Le matériel avait été prélevé sur un Raphicerus compestris tué dans le Kalahari.

Depuis cette époque, le parasıte semble avoir été souvent pris pour Setaria hornbyi (BOULENGER, 1921) que TWAITE considère comme très courant chez les Antilopes africaines (1927) et dont il dénombre treize hôtes divers. Il est vraisemblable, d'après YEH (1959), que TWAITE a eu affaire à plusieurs types de Filaires qu'st a placés - à tort - sous le même nom. Cette erreur par la suite, a été répétée de nombreusés fois et dans ces conditions, l'Incertitude subsiste quant à la répartition exacte de cette espèce.

Artionemo scalprum s'observe au Kenya, en Uganda et en Afrique du Sud (YEH, 1959) chez Raphicerus compestris, Aepyceros melompus, Gazella gronti et Ourebia kenyae.

Les exemplaires du Tchad et de la R.C. A. peuvent être rattachés sans aucune hésitation à $A$. scalprum.

30 Artionema hornbyi (BOULENGER, 1921) N. Comb.

Hôte : Hippotrogus equnus : 7 sur $9(1,6,8,12$, 19).

C'est la plus grande espèce connue d'Artionema A. Hornbyi existe aussi 'en Rhodésie du Nord chez Hippotrogus niger et Alcelophus lichtenstemi (BOULENGER, 1921 ; YEH, 1959), au Transvaal chez Hippotrogus niger et Cobus ellipsiprymnus (MÖNNIG, 1933 b), en Angola (KREIS, 1938) et, sans doute, au Congo ex-Belge (VAN DER BERGHE ef VUYLESTEKE, 1936).

$4^{\circ}$ Artionema bicoronata (VON LINSTOW, 1901) N. Comb.

Hôtes: Damaliscus korrigum : 1 sur 7 (8) Ourebia ourebi splendida : 1 sur 8 (8) Ourebia ourebi dorcas : $\uparrow$ sur 1 (8) Redunca redunco nigeriensis : 2 sur 2

$$
\begin{aligned}
& \text { Redunca redunca : } 1 \text { sur } 1(18) \\
& \text { Gazello rufifrons : } 7 \text { sur } 14(5,15) \\
& \text { Kobus defossa : } 4 \text { sur } B(1,7,8,14) \\
& \text { Adenota kob : } 2 \text { sur } 6(5)
\end{aligned}
$$

Artionema bicoronota est certainement la Filaire péritonéale la plus fréquente des bovidés sauvages du Bassin du Chari.

Comme dans le cas d'A. scalprum. TWAITE (1927) ef MÖNNIG (1933), suivis par d'autres auteurs, ont pris pour Setaria Hornbyi ce qui étalt en réalité $A$. bicoronota (YEH, 1958 ; YEH, 1959).

Une bonne description du parasite a été récemment donnée par YEH (1959) qui note son existence au Mozambıque, en Rhodésie'du Nord, au Tanganyika et au Nyassaland chez Adenoto loderl, Kobus leche, Kobus vardoni et Redunco arundinum. 
$5^{\circ}$ Artionema poultoni (TWAlTE, 1927) N. Comb.

Hôtes: Alcelaphus lelwel : 2 sur $11(4,7)$ ) Domaliscus korrigum : 1 sur 7 (1)

Artionema poultoni a été observé en Uganda chez Bubalus lelwel jacksoni et Damaliscus tiang (TWAITE, 1927) et au Congo ex-Belge chez Domaliscus korrigum jimelo par STRONG et SHATTUCK (1930) et SANDGROUND (1930).

60 Artionemo labiato-papillosa (PERRONCITO, 1882) N. Comb.

Hôte : Syncerus coffer oequinoxialis : 3 sur 5 $(4,20,21)$

Artionema labrato-papillosa est également parasite de Syncerus caffer en Uganda (TWAITE, 1927, DINNIK et Coll, 1963 b) et au Congo exBelge (VAN DEN BERGHE et VUYLSTEKE, 1936).

\section{Gazellofilaria tanganyikae (YEH, 1955)}

Hôte : Gozello dorcas : 1 sur 22 (13)

Autre hôte : Gazella thomsoni (Tanganyika).

Cette curıeuse Filaire a été découverte dans les culs de sac postérieurs du péritoine et dans le triangle de Scarpa. Elle semble rare chez Gazello dorcas.

II s'agit d'un Nématode de grande taille (17 à $22 \mathrm{~mm}$ ), dont la cuticule, finement striée, est couverte de bosses qui se manifestent à $15-18 \mathrm{~mm}$ de l'extrémité antérieure. Les autres caractères anatomiques sont semblables à ceux décrits par YEH (1955 a et b).

Le genre Gazellofilario s'apparente étroitement aux genres Lao et Dirofilaria, la différence tenant à la présence de bosses cuticulaires dans le premier cas. Le genre Dirofilaria comprend deux espèces parasıtes de Cephalophes: $D$. kuelz" (RODENWALDT, 1910) au Congo ex-Belge (GEDOELST, 1916) et D. asymmetrica (KREIS, 1938) en Afrıque du Sud. CHABAUD ef ROUSSELOT $(1956, a)$, d̀ partır d'exemplaires de Cephalophus casteneus dorsalis, mettent en synonymı les deux espèces précédentes.

YEH (1955 b), du fait de l'absence de bosses cuticulaires chez $D$. kuelzıi, hésıte à grouper les trois Nématodes sous le nom de Gozellofilaria kuelzii (RODENWALDT, 1910).

\section{III. - Les helminthes de l'appareil vasculaire}

10 Schistosoma bovis (SONSINO, 1876).

Hôtes : Adenoto kob : 1 sur 6 (5)
Hippofragus equinus : 1 sur $9(8)$

Kobus defosso : 1 sur $8(7)$

Autre hote : Limnotragus speker gratus - Congo

ex-Belge (VAN DER BERGHE, 1943).

\section{IV. - Les helminthes des muscles ef du fissu conjonctif intermusculaire}

10 Cysticercus bovis (COBBOLD, 1866)

Hotes : Gazello rufifrons : 1 sur 11 (15)

Adenota kob: 1 sur 6 (9)

Syncerus caffer: 1 sur 5 (18)

Autre hôte: Oryx sp. (TAYLOR, 1958).

$2^{\circ}$ Cysticercus dromedarii (PELLEGRINI, 1945).

Hôtes : Damolıscus korrigum : 3 sur 7 (19)

Kobus defosso: 1 sur 8 ( 8 )

Gazello rufifrons : 2 sur 11 (14 ef 15)

Hippotrogus equinus : 1 sur 9 (8)

Alcelaphus lelwel : 1 sur 11 (7)

Redunco redunco nigeriensis : 1 sur $2(8)$

Toux moyen d'infestation des Antilopes par C. bovis et C. dromedaru: environ 15 p. 100 dont 11 p. 100 por C. dromedaril.

Cysticercus dromedoril est le Cysticerque de Toenia hyoenoe (BAER, 1924), Cestode de Hyoena crocuta et de Hyaena hyoena au Soudan (BAER, 1923), en Afrique du Sud (BAER, 1926), au Tanganyika (BAYLIS, 1937), au Kenya, en Somalie où 70 p. 100 des Hyènes sont atteintes (PELLEGRI. NI, 1950), au Congo ex-Belge (BAER et FAIN, 1955) et au Tchad (GRABER, 1959) où $T$. hyoenae infeste 9 hyènes sur 10 dans l'Est du Territore.

Le Cysticerque se présente sous la' forme d'un kyste ovoìde de 5 à $20 \mathrm{~mm}$ au milieu duquel on distingue un scolex globuleux de 600 à $1.000 \mu$ portant quatre ventouses et armé d'une double couronne de crochets (32 à 44). Les plus grands mesurent de 187 à $218 \mu$ (206 $\mu$ pour un exemplaire de Gozella rufifrons) et les plus ipetits $130 \mu$.

Le diagnostic différentiel est difficile dans les pays où Cysicercus cellulosae du porc est abondant : dans ce cas, il faut tenir compte de caractères tels que le plus petıt nombre de crochets (22 à 31), la longueur des plus grands $(160-180 \mu$ ) et leur aspect (manche plus court que la lame).

Cysticercus dromedaris a été soupçonné par MARTINAGLIA en 1932 à Johannesburg. Il a été recueilli par PELLEGRINI (1947) $a, b, c, d)$ en Somalie chez le chameau et chez le bceuf et par COCEANI (1949) et CALL (1949) en Erytrée 
chez les mêmes animaux et chez Cephalophus grimmia. URQUARTH ef ZAPHIRO (1960), au Kenya, retrouvent, chez Gorgon raurnus, Gazello gronti et Gazella thomsoni, un Cysticerque armé dont la forme adulte — supposent les auteurs pourrait être Taenia hyaenae de la Hyène.

\section{V. - Les helminthes du foie ef des canoux biliaires}

10 Fasciola gigantica (COBBOLD, 1855).

Hôtes : Adenota kob : 1 sur 6 (12) Syncerus caffer : 1 sur $5(18)$.
Autres hôtes: Alcielophus, Kobus defassa, Adenoto kob Congo ex-Belge (STUNKARD, 1929)

20 Stilesia hepatica (WOLFFHÜGEL, 1903).

Hôtes: Redunca redunca nigeriensis : 1 sur 2 ( 8 ) Hippotragus equinus 7 sur $9(1,6,8$,

$12,19)$

$$
\text { Kobus defassa: } 5 \text { sur } 8(7,8,12)
$$

Ces Cestodes sont parfois très nombreux (plusieurs dizaines de grammes) dans les canaux biliaires.

Stilesia hepatico est un Cestode hépatique courant des grandes Antilopes africalnes: (tableau $V$ ).

TABLEAUU $\mathbb{N}^{\circ} \mathrm{V}$

\begin{tabular}{|l|l|c|c|}
\hline \multicolumn{1}{|c|}{ Pays } & \multicolumn{1}{|c|}{ Espèces animales } & Auteurs & Date \\
\hline Pays Masai & Tragelaphus seriptus & Furhmann & 1909 \\
Afrique du Sud & Sylvicapra grimui transvaglensls & Wolffhttgel & 1903 \\
& Hippotragus equimus & - & - \\
Afrique du Sud & Cephalophus & Gough & 1910 \\
Afrique de l'Est & Hippotragus equinus & - & - \\
Kegya & Hippotragus sylvaticus & - & - \\
Ethiopie & Kobus kob & Southwell & 1929 \\
Congo er-Belge & Gazella granti laculum & Furhmern et Baer & 1943 \\
Dahomey & Kobus defassa crawshayi & Heqer et Fain & 1955 \\
& Hippotragus equinus & Norel & 1959 \\
\hline
\end{tabular}

$3^{\circ}$ Crossofaenia baeri (MAHON, 1954).

Hôte: Trogelophus scriptus : 1 sur 1 (17).

Voisin des genres Wyominia ef Thysanosoma qui sont américains, le genre Crossotaenio, inventé par MAHON (1954) pour un Cestode hépatique de Cephalaphus sylvicultor du Congo ex-Belge, est caractérisé par le bord plissé des segments, l'absence de vésicules séminales externes ef internes, la position des conduits génitaux par rapport aux canaux excréteurs et l'aspect de l'utérus.

Comme au Congo, les exemplaires de R. C. A. se présentaient sous l'apparence de fragments grisâtres sans scolex. La largeur du Cestode esł d'environ 4 à $5 \mathrm{~mm}$. Les dessins figurés par MAHON (1954) et les coupes sériées pratıquées en divers points du Crossotaenia de Tragelaphus coïncident.

A noter également la présence à peu près certaine de ce Cestode dans les collections du Dr ROUSSELOT à Brazzaville. L'hôte en était Cephalophus dorsalis castaneus.

\section{$4^{\circ}$ Linguatula nuttali (SAMBON, 1922).}

Hôte : Hippotragus equinus : 1 sur 9 (8).

Dans le tissu hépatique de cette Antılope, 60 formes immatures ont été prélevées. D'aspect linguiforme, élargie à son extrémıté antérieure et rétrécie postérieurement, la nymphe mesure 6,5 à $7 \mathrm{~mm}$. II existe de 122 à 127 segments. Les quatre crochets, en forme d'arche, sont de taille inégale : 542 à $613 \mu$ pour les crochets internes et 626 à $672 \mu$ pour les crochets externes. En outre, l'extrémité postérieure est fourchue, presque bilobée.

Ces caractères incitent à penser qu'il s'agit là de formes nymphales de Linguatula nuttali, décrit par SAMBON (1922) à partir de Pentas- 
tomidés récoltés dans les cavités nasales de Félis leo en Afrique Orientale. SAMBON (1922), HEYMONS (1935) ef FAIN (1961) sont d'avis que les Nymphes de Linguatules trouvées chez diverses Antilopes et assimilées à Linguatula serrata sont en réalité des $L$. nuttali.

D'ailleurs, les formes nymphales des deux espèces sont différentes. Nous avons eu la possibilité d'établir la comparaison entre les exemplaires d'Hippotragus equinus et d'autres recuellis chez Bibos indicus à Abéché et Bos tourus en France. Pour Linguatula serrata, la taille n'est pas la même $(4,7$ à $5 \mathrm{~mm})$; les segments sont au nombre de 87-90; les quatre crochets, en forme d'arche sont sub-égaux ( 414 d̀ $450 \mu$ ) et l'extrémité postérieure est simplement fendue.

\section{C. - RÉFLEXIONS \\ SUR LA DISTRIBUTION GÉOGRAPHIQUE DE CES HELMINTHES}

Les Helminthes des bovidés ef des suidés sauvages du Bassin Logone-Chari comportent donc des espèces très largement distribuées dans toute I'Afrique du Sud du Sahara, que ce soit d'Est en Ouest ou du Nord au Sud. C'est le cas notamment de Poramphistomum microbothrium, Cotylophoron cotylophorum. Colicophoron calicophorum, Cormyerius spatiosus, Haemonchus contortus, Stilesia globipunctota, Gostrodiscus aegyptiacus, Buck leyuris globuloso, les Oesophagostomes des Phacochères, Artionema hornbyi, Artionemo poultoni, Artionema congolensis, Cysticercus bovis et Fascoila gigantica, Stilesia hepatica.

D'autres espèces ont été revues ça et là en divers points d'Afrique Norre : Stephanopharynx compactus, Moniezı monardi, Agriostomum cursoni, Bunosiomum dentatum, Artionema scalprum, Artionema bicoronato.

D'autres sont encore plus rares et n'ont été signalées qu'une fois ou deux : Gozellofilaria tonganyikae, Cysticercus dromedarii, Longistrongylus meyerı, Avifellina sandgroundi, Avitellino woodlandi, Linguatulo nuttali. Cormyerius endopopillatus.

D'autres enfin, - jusqu'à plus ample informé — semblent propres à la cuvette Tchadienne : Carmyerius papillatus et Cormyerius parvipapillatus (Tchad, R. C. A.), s'il a fait abstraction du Kobus defassa tué en 1957 au Zoo de Brazzaville dont l'origine exacte est en réalıté indéterminée (Tchad ? Congo ?) et sur lequel ont été recueillis les prototypes des deux espèces précédentes.

Dans l'ensemble, la faune parasitaire du Bassın du Chari ne paraît pas pouvorr être dissociée de celle des bassins du Congo du Nil, les communications se faisant au Sud par l'intermédiaire de l'Oubangui et, à l'Est, par le Dar-Four ef le Bahr-el-Ghazal.

C'est ce qu'avait remarqué STUNKARD (1929) à propos de Gastrodiscus aegyptiacus et DOLLFUS (1950) écrivalt : « La faune tropicale trématodologique de l'Afrique tropicale et subtropicale paraît assez homogène ; les espèces décrite) d'abord de la vallée du Nil, du Soudan ou de I'Uganda sont peu à peu retrouvées vers l'Ouest jusqu'à l'Atiantique et vers le Sud jusqu'à l'Union Sud-Africaine, chez les mêmes hôtes ou chez des hôtes vicariants ». Ce qui est vrai des Trématodes, l'est également des Cestodes, des Nématodes ou des Pentastomidés.

A cet égard, on ne dolt pas sous-estimer le rôle des migrations de gibier dans la dissémination d'espèces parasites spécifiques que l'on retrouve parfols en des points fort éloıgnés les uns des autres.

\section{D. - ROLE PATHOGÈNE}

II est à peu près totalement inconnu.

II est bien évident que les Helminthes comme les Carmyerius, les Gostrodiscus, les Agriostomum, les Bunostomum, les Haemonchus, les Distomes du fore et les Cestodes dont on connaît l'incıdence sur les animaux domestiques sont susceptibles d'exercer une action similaire chez les Bovidés sauvages. L'atteinte parasıtare n'est cependant pas facile à déceler et, sur les cent Artiodactyles autopsiés, aucun cas clinique d'Helminthiase n'a pu être mis en évidence.

Il est probable que, de par leur mode de vie, les Antilopes ef les Suidés ont une alımentation plus riche, plus abondante que les animaux domestiques et que, dans ces conditions, l'équilibre hôte-parasıte est à peu près réalısé : c'est ce qui se passe pour les Gazelles corinne qui utilisent de préférence un pâturage arbustıf ou pour les Gazelles dorcas qui vivent sur les grandes dunes du Nord.

En outre, les transhumances de certaines Antilopes - souvent sur de très longues distances - 
les préservent de toute disette grave ef la consommation de Cucurbitacées, d'Euphorbiacées, d'herbes, vertes purgatives ou de sels minéraux dovés d'un certain pouvoir anthelminthique (natron) tend à réduire le nombre de parasites intestinaux.

La création de parcs, s'ils ne sont pas suffisamment vastes et avec trop d'animaux par unité de surface, risque d'entraîner une rupture d'équilibre avec développement concomitant du parasitisme et mortalıté élevée. Dans ces pays, le fait est

Waterbuck $n^{0} 1$

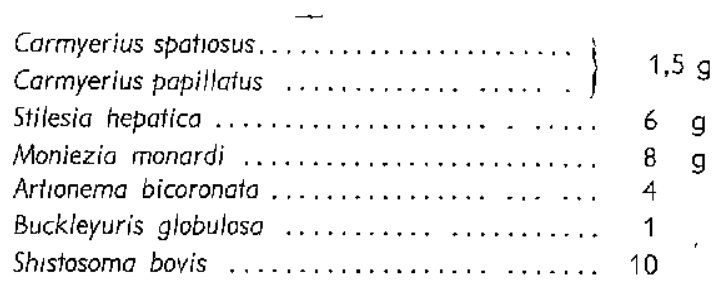

bien connu, chez le mouton, dès que l'on dépasse une centaine de têtes et, dans une moındre mesure, chez le zébu. Il se'produit peut-être le même phénomène pour les ruminants sauvages et ce serait-là, entre autres, l'explication de la raréfaction relative des Bubales du Tchad depuis quelques années.

A noter également, le grand nombre d'associations parasitarres. Ce sont de loin des Waterbucks qui sont les Antilopes les plus parasitées:

\section{Waterbuck $\Pi^{\circ} 2$}

Poramphistomum microbothrium. . ..........

Carmyerius parvipapiliatus $\ldots \ldots \ldots \ldots \ldots \ldots$.

Cormyerius spatiosus ..................

Stephanopharynx compactus ............ $2 \mathrm{~g}$

Cysticercus dromedarii ................. 3

Artionemo bicoronota .................. . 15

Cooperia punctato .................. 150

Chez les Hippotragues, les associations peuvent être de divers types :

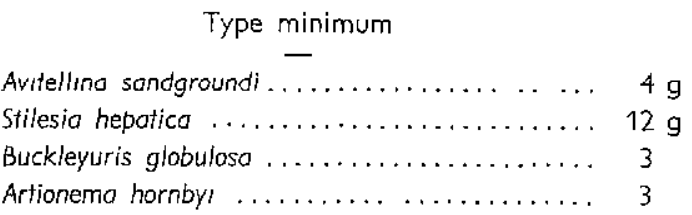

Type maximum

Globulosa Buckleyuris ............... $15 \mathrm{~g}$

Shistosomo bovis ................... 2

Bunosiomum dentatum ................ 10

Cysticercus dromedorii .... .............. 4

Moniezia monordi ... .................. $20 \mathrm{~g}$

Stılesı hepotica .................... $13 \mathrm{~g}$

Les infestations des Bubales ef des Damalisques sont plus discrètes et dépassent rarement quatre éléments :

Bubale no 1

Cotylophoran cotylophorum .............. $7 \mathrm{~g}$

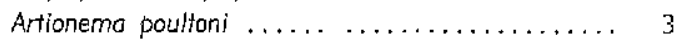

Longistrongylus meyeri $\ldots \ldots \ldots \ldots \ldots \ldots \ldots$

Les petites Antilopes, telles que les Reedbucks, les Ourébis ou les Cobs de Buffon paraissent assez peu parasitées: le maximum est de trois espèces parasites différentes par animal. II en est de même chez les Gazelles darcas où le polyparasitısme à quatre éléments constitue un maximum.

\section{E. - LES ARTIODACTYLES SAUVAGES SONT-ILS DES RÉSERVOIRS DE PARASITES POUR LES ANIMAUX DOMESTIQUES QUI VIVENT DANS LEUR VOISINAGE ?}

Dans les conditions de la Républıque du Tchad et de la R. C. A., il existe - semble-t-il — :
Bubale $n^{0} 2$

Longistrongylus albifrontis ............. 5

Avitellina sandgroundi................. $3 \mathrm{~g}$

10 Des helminthes obsolument spécifiques des bovidés et des porcins sauvages.

Ce sont :

Carmyerius exoporus.

Carmyerius endopapillatus.

Moniezia monardi.

Avitelimo sandgroundi.

Crossotaenia baeri.

Longistrongylus meyeri.

Longistrongylus olbifrontis.

Kobusinema schrenki.

Haemonchus vegliai.

Parabronema skrjabini.

Ascaris phocochoeri. 
Agriostomum cursoni.

Bunostomum dentatum.

Daubneyia m'wanzee.

Daubneyia oldi.

Daubneyia rouboudi.

Pygarginema africono.

Moniezia mettamı.

Murshidia pugnicaudoto.

Artionemo congolensis.

Artionemo scalprum.

Artionema hornbyi.

Artionema poulton.

Gazellofilaria tanganyikae.

Linguatula nuttali.

Ces Helminthes n'ont - pour l'instant - jamaıs été rencontrés chez les animaux domestiques de l'Afrique centrale.

$2^{\circ}$ Des parasifes qui, s'ils sont assez fréquents chez les artiodactyles sauvages, sont beaucoup moins bien représentés chez les ruminants domestiques:

Rentrent dans cette catégorie les Helmınthes suivants :

a) Calicophoron colicophorum.

b) Cotylophoron cotylophorum.

c) Stephanopharynx compoctus.

d) Carmyerius spatıosus.

e) Cormyerius papillatus.

f) Carmyerius parvipapillatus.

TABLEAT HO UI

\begin{tabular}{|c|c|c|c|c|c|c|}
\hline \multirow[b]{2}{*}{ Espècen parasitées } & \multicolumn{3}{|c|}{ Nombre d'animaux autopeiés } & \multicolumn{3}{|c|}{ Pourcentage d'an1maur parasités } \\
\hline & $\begin{array}{c}\text { Bovins } \\
\text { domestiques }\end{array}$ & $\begin{array}{c}\text { ovins } \\
\text { domestiques }\end{array}$ & $\begin{array}{l}\text { Bovidés } \\
\text { seuvages }\end{array}$ & $\begin{array}{c}\text { Bovins } \\
\text { domestiques }\end{array}$ & $\begin{array}{c}\text { Oving } \\
\text { domestiques }\end{array}$ & $\begin{array}{l}\text { Bovidés } \\
\text { sauvages }\end{array}$ \\
\hline Cotylophoron cotylophorm & 4.965 & 3.741 & 75 & 1,1 p. 100 & $2,1 \mathrm{p} \cdot 100$ & $5.3 \mathrm{p} .100$ \\
\hline Cotylophoron calloophorum ${ }^{+}$ & - & - & - & $0,2 \mathrm{p} .100$ & 0,2 p. 100 & $2,6 \mathrm{p} \cdot 100$ \\
\hline Stephenopharyms compactus & - & - & - & $0,1 \mathrm{p} \cdot 100$ & 0 & $5,3 \mathrm{p} \cdot 100$ \\
\hline Carmyerius apatiosus & - & - & - & 3,5 p. 100 & $1,1 \mathrm{p} .100$ & $12 \quad \mathrm{P} \cdot 100$ \\
\hline $\begin{array}{l}\text { Carmyerius papillatus } \\
\text { Cacmyerius parvipapillatus }\end{array}$ & - & - & - & 0,4 p. 100 & $0.1 \mathrm{p} \cdot 100$ & $6,6 \mathrm{p} \cdot 100$ \\
\hline Stilesia hepatica & - & - & - & 0 & $1,9 \mathrm{p} \cdot 100$ & $16,4 \mathrm{p} .100$ \\
\hline Cysticercus dromedaril & - & - & - & 0,1 p. 100 & 0 & 11,3 p. 100 \\
\hline
\end{tabular}

+ - Le fait n'est valable que pour le Thahad. C. Cotylophorm est, en R.C.A., le Paramphistone dominant des ruminants domestiques (8 sur 10). P. microbothrium est rare.

g) Stilesia hepatica.

h) Cysticercus dromedarii.

Le tableau no VI donne, pour le Tchad, les pourcentages d'infestation chez les bovins et les ovins domestiques d'une part, et chez les Bovidés sau'vages d'autre part.

II apparaît donc très nettement que certains de ces Helminthes sont plutôt des parasites de ruminants sauvages que de ruminants domestiques. C'est le cas de Cysticercus dromedarm, de Stephanophorynx compactus, de Cormyerius papillatus, de Cormyerius parvipapillatus et de Stilesio hepotıca, cestode à propos duquel GOUGH (1911) écrivait déjà :

« II est probable qu'à l'origine S. hepatica était un parasite des ruminants sauvages et on peut supposer qu'il s'est adapté secondarement au mouton. Le fait qu'il ne soit pas fait mention du parasite dans les autres parties du monde, sa présence chez les antilopes, sı caractérıstique en Ethiopie, ses nombreux hôtes, donnent à penser qu'au commencement il n'était pas parasıte du mouton. »

La répartition de ces Helminthes, tant chez les 


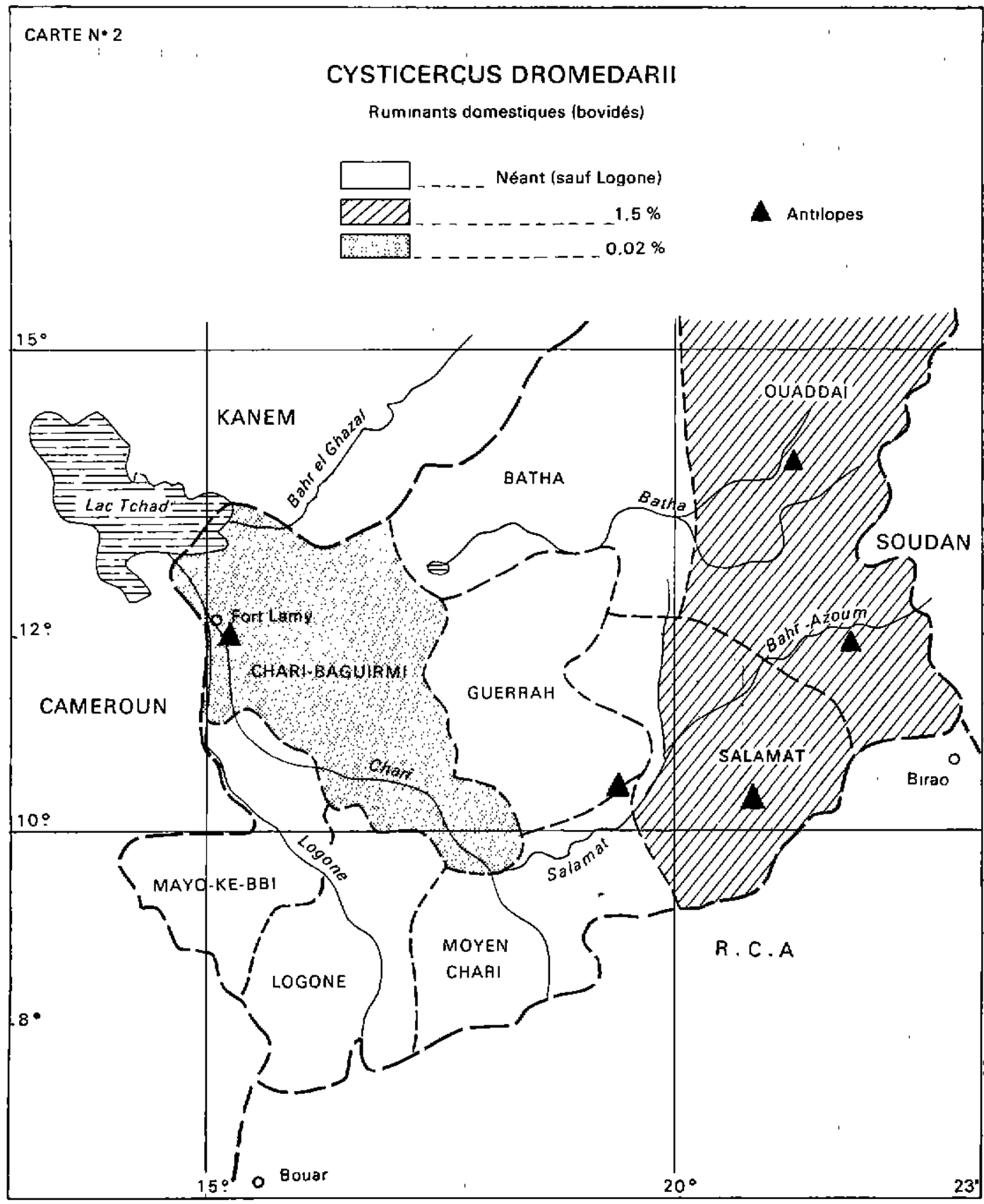




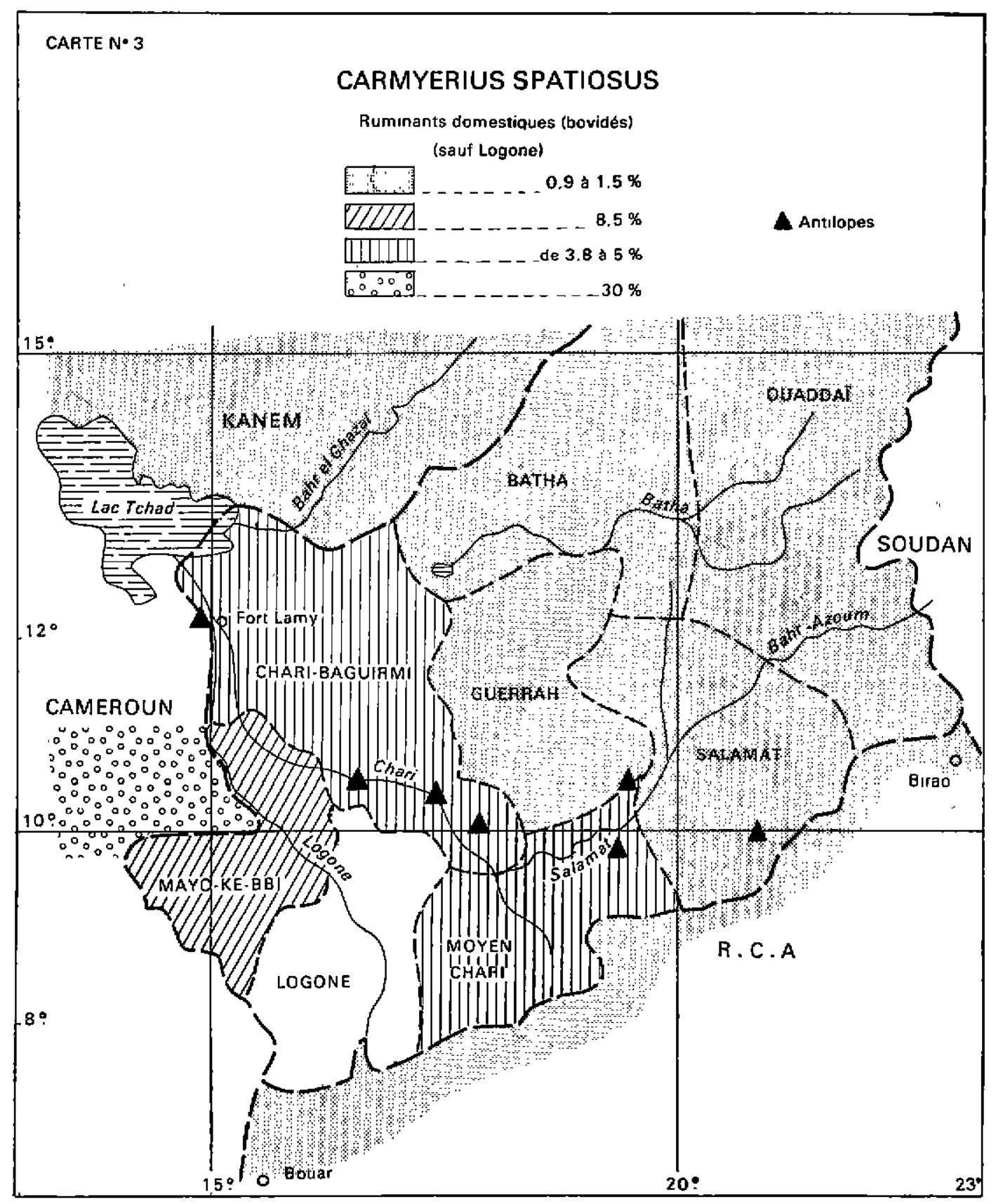




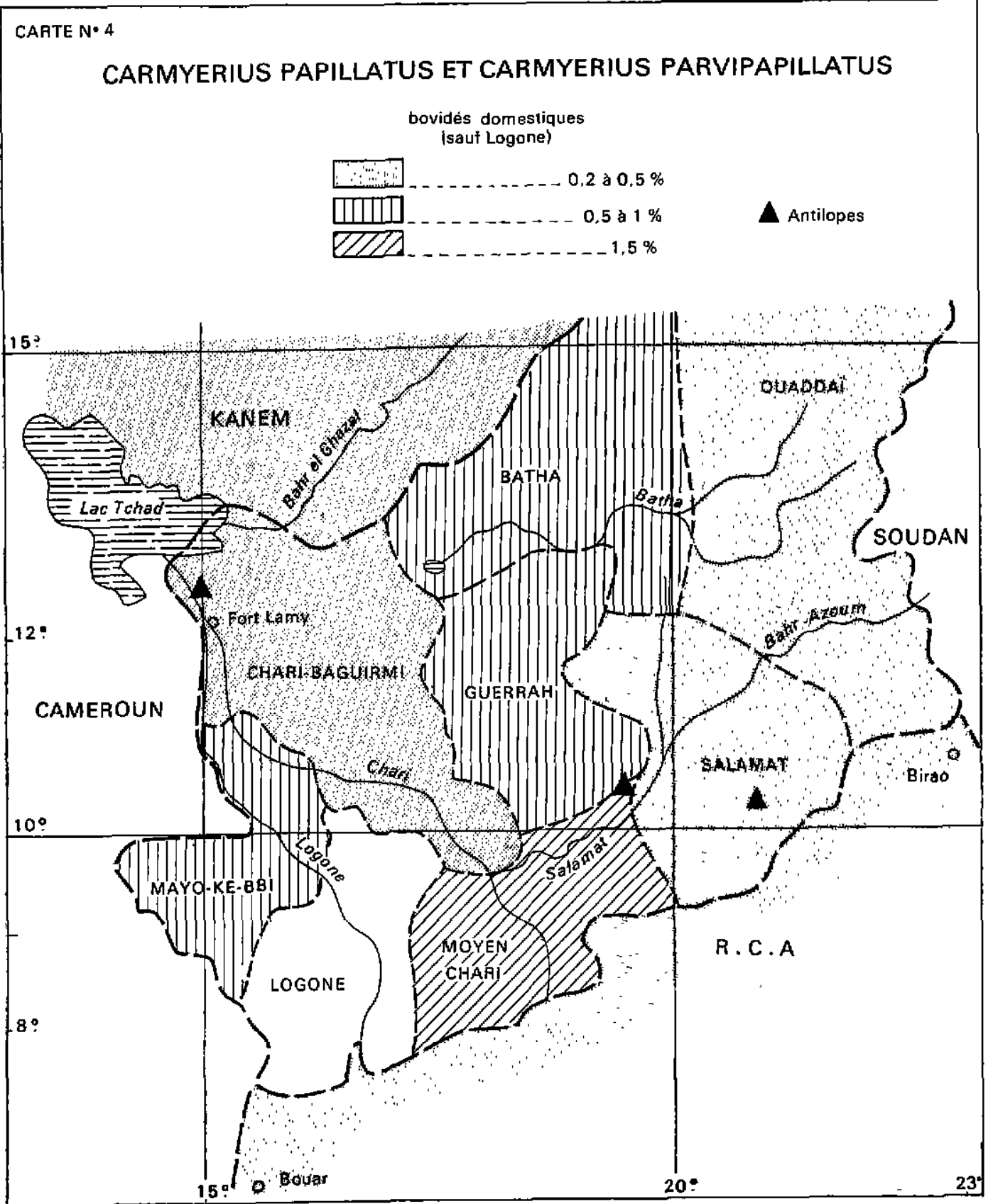




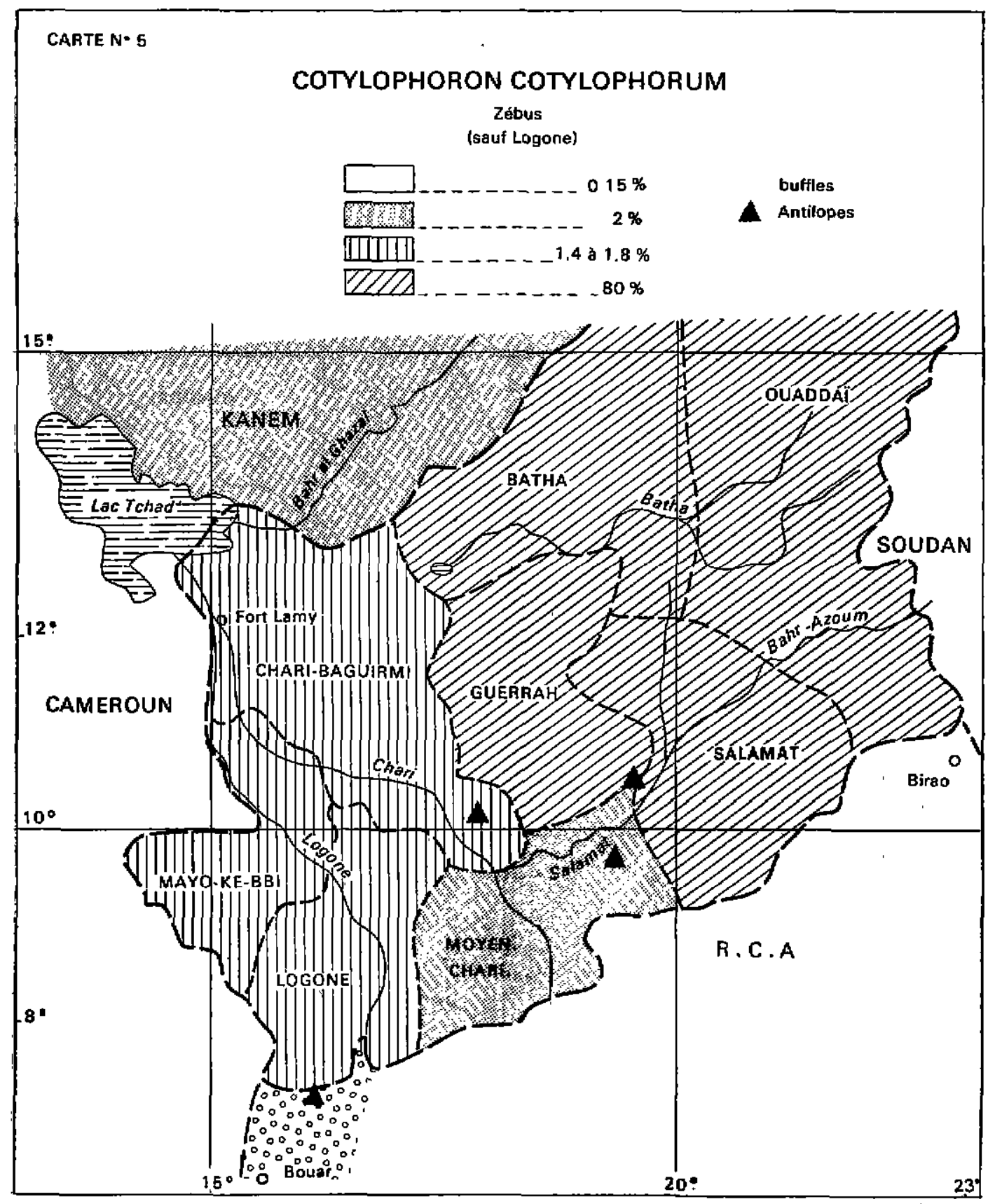




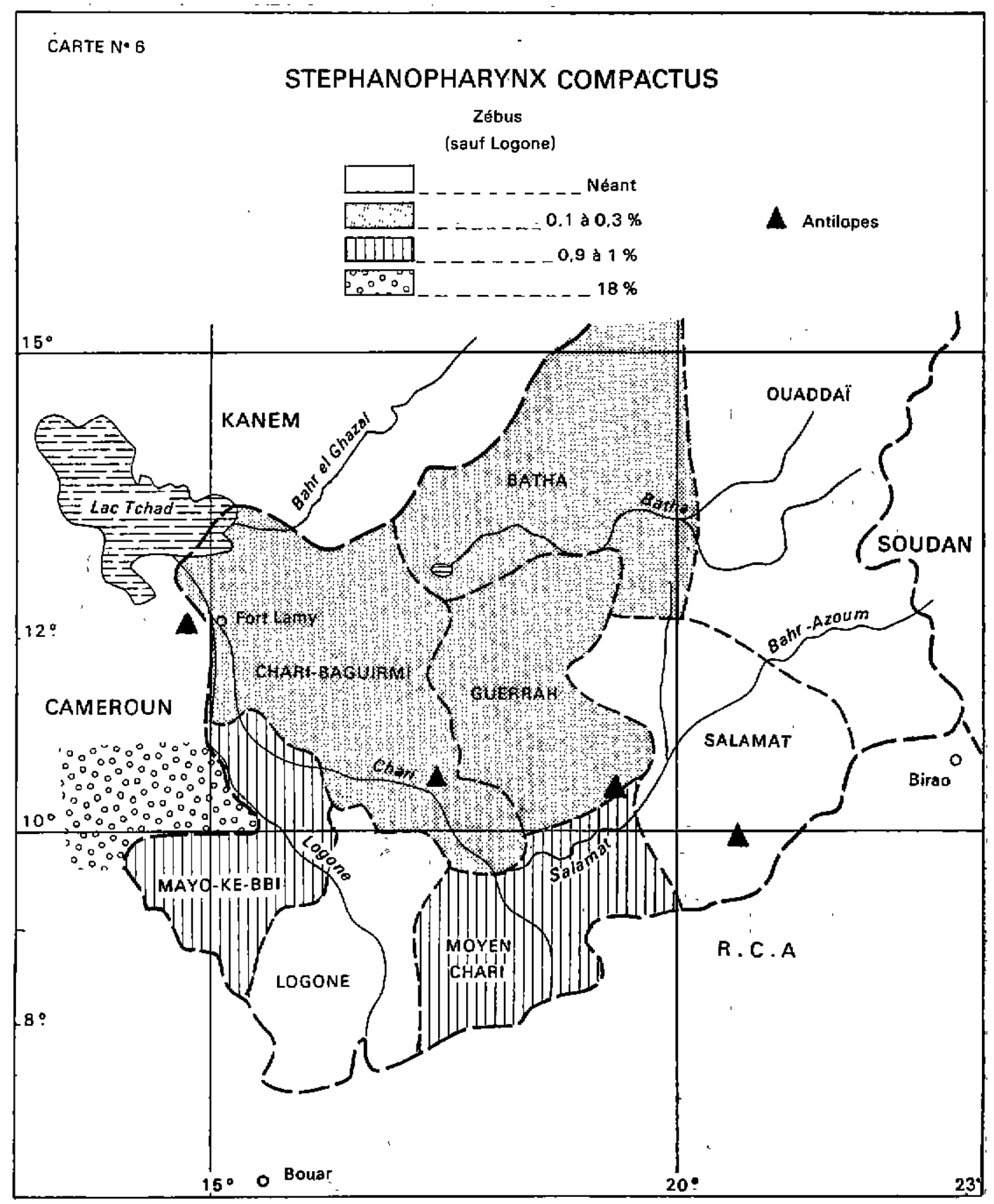




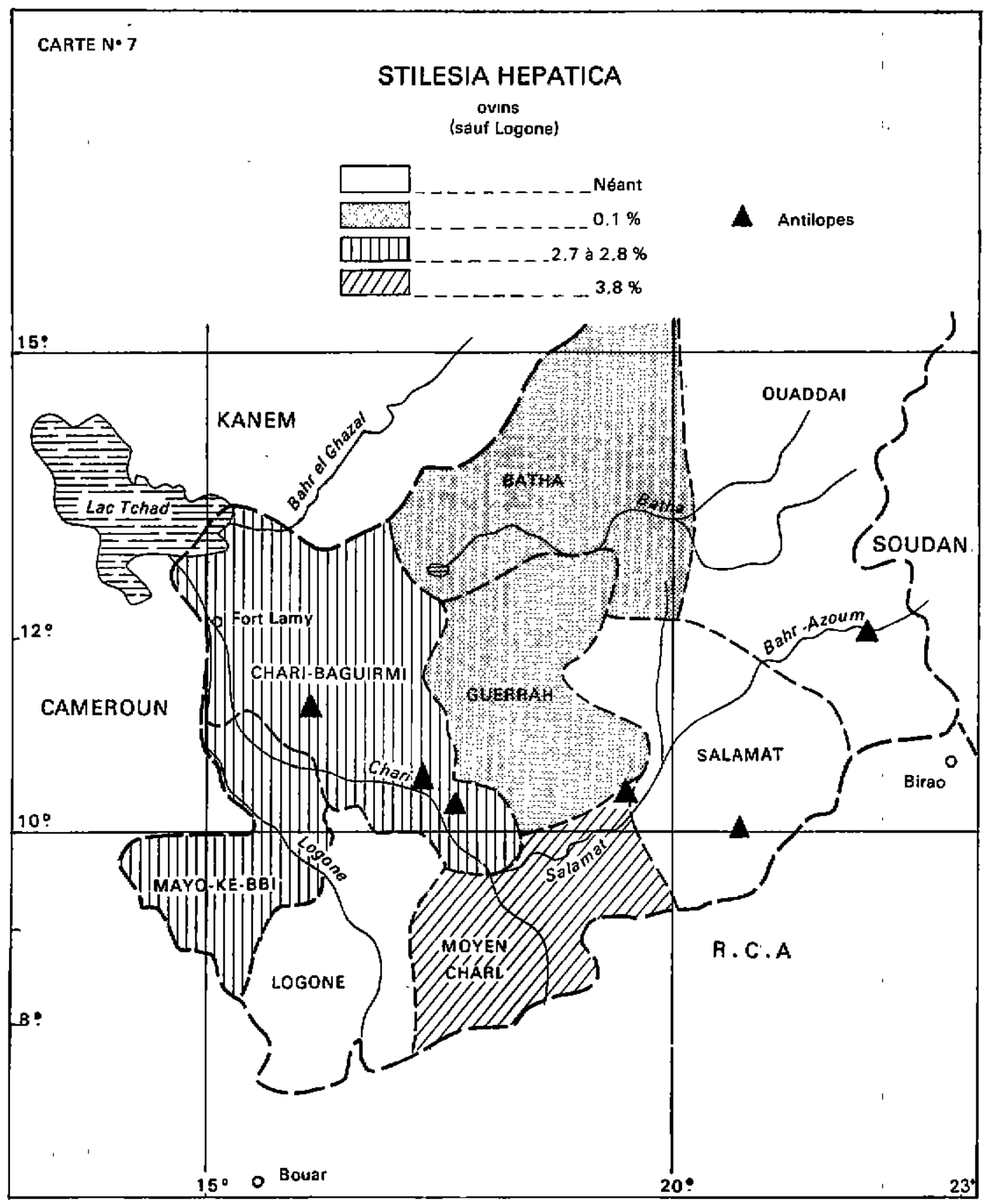


bovidés domestiques que chez les bovidés sauvages, est figurée sur cinq cartes annexes (2 à 7 ) valables pour l'ensemble du Tchad.

En définitive, les huit parasites en question ne se retrouvent, chez les animaux domestiques, que lorsqu'il y a rencontre, sur les mêmes parcours, entre faune domestique et faune sauvage : le fait se produit, dans l'Est et le Centre tchadien, à l'accasion de la descente ou de la remontée des troupeaux nomades.

Dons ce cas, les Artiodactyles sauvages servent indirectement de réservoirs de parasites, mais leur rôle semble au demeurant limité, quand on considère le petit nombre d'animaux domestiques qui hébergent ces parasites.
$3^{0}$ Des espèces très abondanfes chez les animaux domestiques ef beaucoup plus rares chez les artiodactyles sauvages.

Voir tableau no VII.

Le tableau no $V$ ll est accompagné de cinq cartes ( 8 à 12) intéressant la distribution géographique de Paramphistomum microbothrium, Haemonchus contorfus, Fasciola gigantica, Schistosoma bovis et Gastrodiscus aegyptiocus.

Comme dans le cas précédent, les mélanges de populations animales favorisent la dispersion de ces Helminthes à l'intérieur de groupes zoologiques différents.

TARIRAT I* YII

\begin{tabular}{|c|c|c|c|c|c|c|c|c|}
\hline & \multicolumn{3}{|c|}{ Manbre d'anianx autopsidis } & \multicolumn{5}{|c|}{ 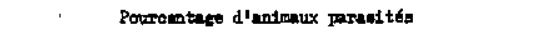 } \\
\hline & $\begin{array}{c}\text { Berins } \\
\text { donestiques }\end{array}$ & $\begin{array}{c}\text { Orinte } \\
\text { doneatiques }\end{array}$ & $\begin{array}{l}\text { Boriass } \\
\text { Exraget }\end{array}$ & $\begin{array}{r}\text { Bo } \\
\text { domea }\end{array}$ & $\begin{array}{l}\text { Hins } \\
\text { tiques }\end{array}$ & $\begin{array}{c}\text { Orins } \\
\text { donestiques }\end{array}$ & $\begin{array}{l}\text { Borldés } \\
\text { emvaser }\end{array}$ & $\begin{array}{c}\text { Anes } \\
\text { Chovarir }\end{array}$ \\
\hline Paramphtetonum mierokrthrilum & 4.965 & 3.741 & 75 & 18 & p. 100 & $20 \quad$ p.100 & 16,2 p.100 & \\
\hline Hacuogolns sontortus & - & - & - & & • & $22,8 \quad$ & $16,2 \quad "$, & \\
\hline Shistomome boril & - & - & - & 25 & • & 10,4 & 4 & \\
\hline Fascioln oleastion & - & - & - & 22 & $\bullet$ & - ' & 2,7 & \\
\hline Cyoticerone bovis & - & - & - & & " & 0,05 & 4 & \\
\hline Stilesis globlpomoteta & - & - & - & 0,01 & $"$ & 32 & 1,3 & \\
\hline Desophagostoum aoluxbingum +4 & - & - & - & 0 & $"$ & 35 & 2,6 & \\
\hline Artioneas labieto-paptlloes & - & - & - & 25 & $n$ & $0,01 \quad$ & 1,3 & \\
\hline Buciclesurite slobuions & - & - & - & 5 & $n$ & $3, \cdots$ & 9.3 & \\
\hline Cooperile punotatn & - & - & - & 30 & $n$ & D & 1,3 & \\
\hline Avitellina woodladid & - & - & - & 0 & $n$ & 3,5 & 2 & \\
\hline 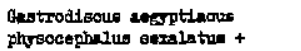 & $40 \mathrm{p} .100$ & des Phnogohbritad & peraaltes & & i & & & $92 \mathrm{p}+100$ \\
\hline
\end{tabular}

+ perancil to du poro dousatil que

+ Graber ot Hecereur (1956)

\section{CONCLUSIONS}

Jusqu'à plus ample informé, 26 espèces d'Helmınthes absolument spécifiques ont été dénombrées chez les Artiodactyles sauvages de la R. C. A. et de la République du Tchad.

21 autres sont communes aux Bovidés ef Suidés sauvages et aux animaux domestiques : huit d'entre elles sont plutôt des parasıtes d'Antilopes qui passent chez le zébu ou le mouton, à la faveur des transhumances et des brassages de populations anımales. Les treize autres sont des espèces très courantes, à large dispersion et fortement implantées dans le pays.

Il est donc difficile d'affirmer que les animaux de la faune africaine représentent, en matière d'Helminthiases, un danger certain pour les bovins, ovins, caprins ou camelins qui vivent à leur contact ou dans leur voisinage.
Leur rôle ne doit pas, non plus, totalement être sous-estimé, car ils sont susceptibles - souvent et assez massivement - d'héberger des Helminthes aussi dangereux pour le bétail qu'Haemonchus contortus, Carmyerius spotiosus, Gostrodiscus aegyptiacus, Oesophogostomum columbianum, Cooperia punctata, Schistosoma bovis, Fasciola gigantica, Stilesia globipunctata ou Stilesia hepatica. C'est ainsi que, dans les régıons Nord du Tchad, les Gazelles dorcos semblent être, en salson sèche, le principal réservoir d'Haemonchus contortus, nématode qui, lors des premıères chutes de pluies, ira infester les moutons, les chèvres et les jeunes zébus qui utilisent les mêmes parcours.

Il importe donc, dans les estimations ef les projets d'érodication des Helminthiases, de tenir 


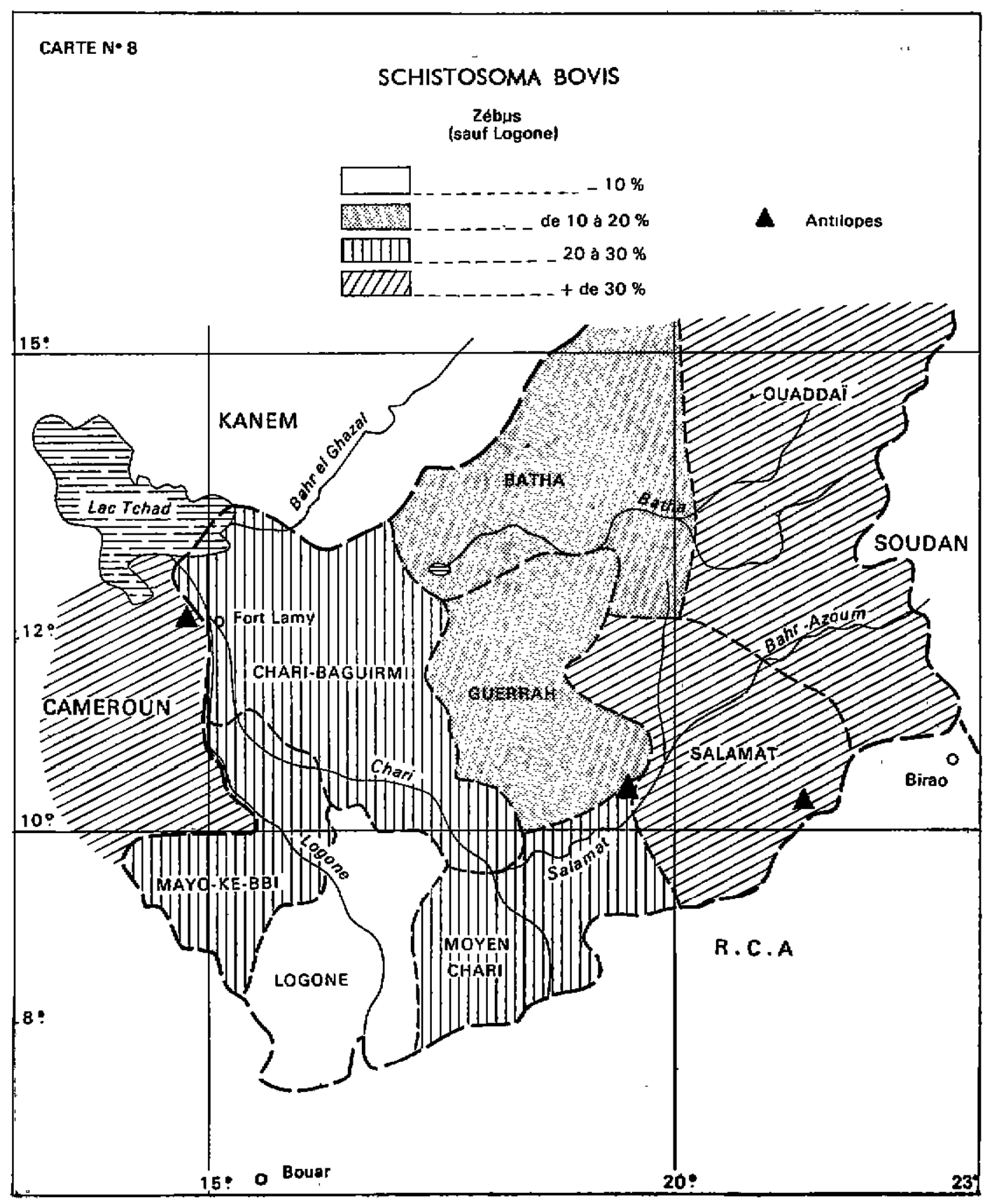




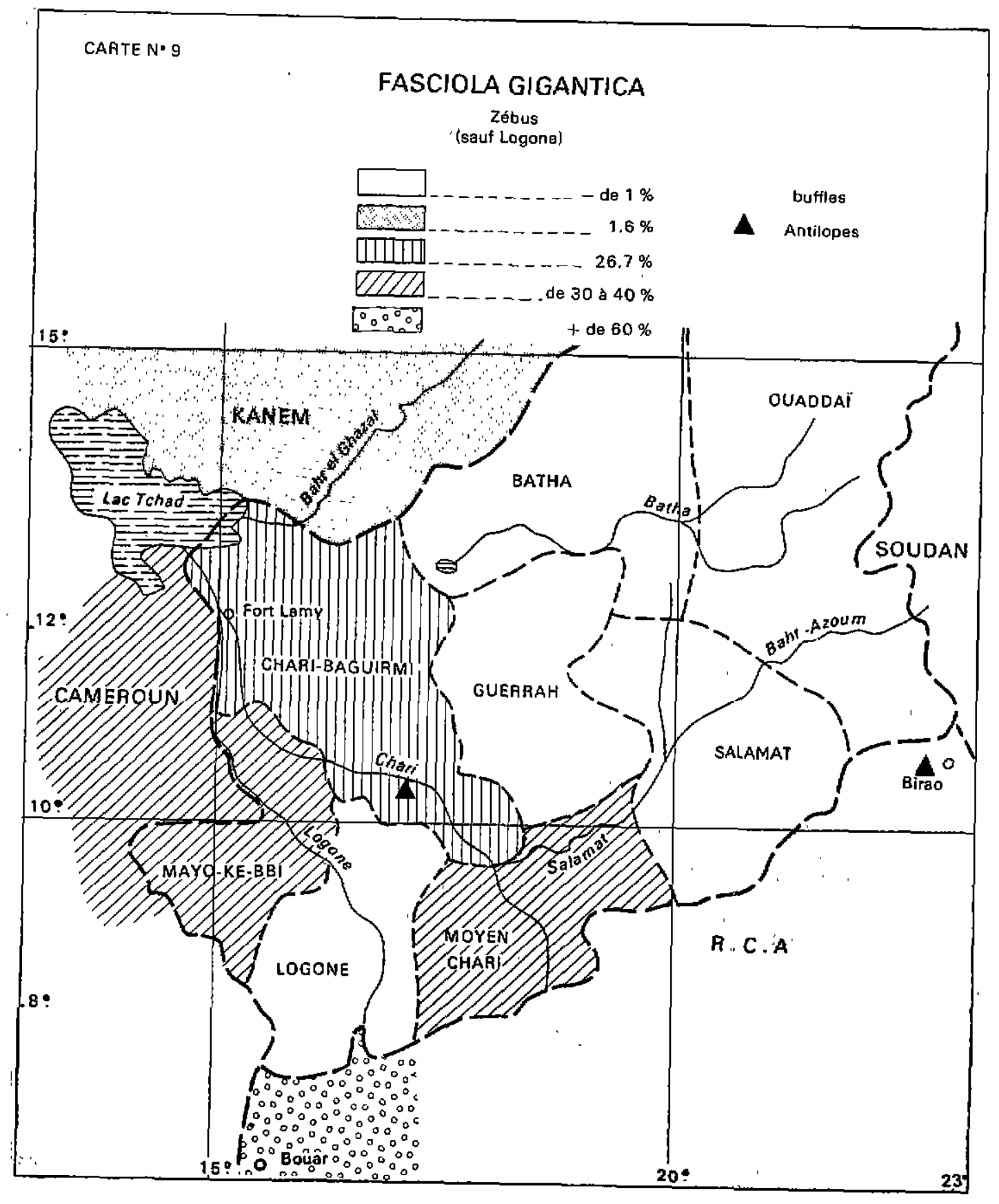




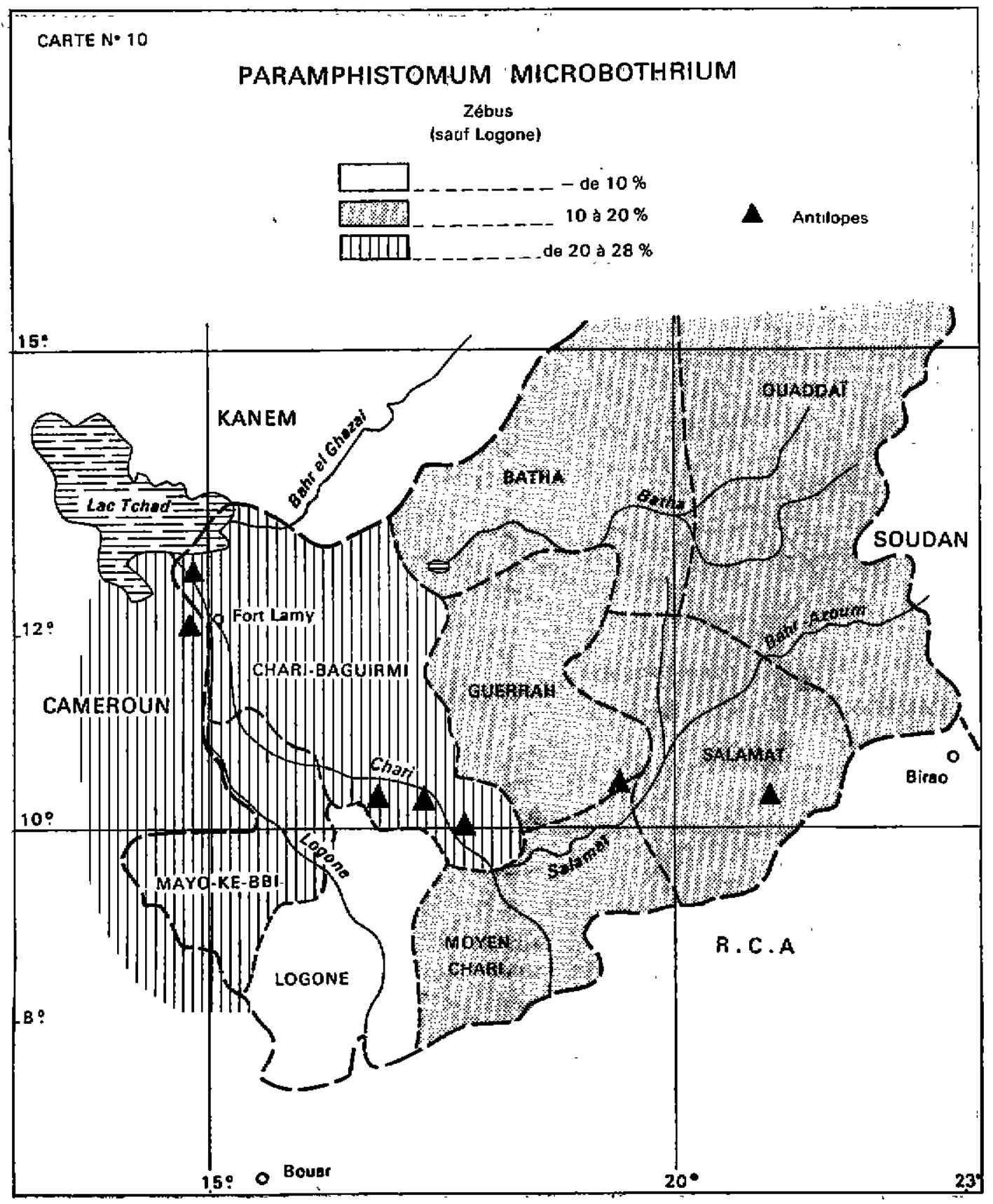




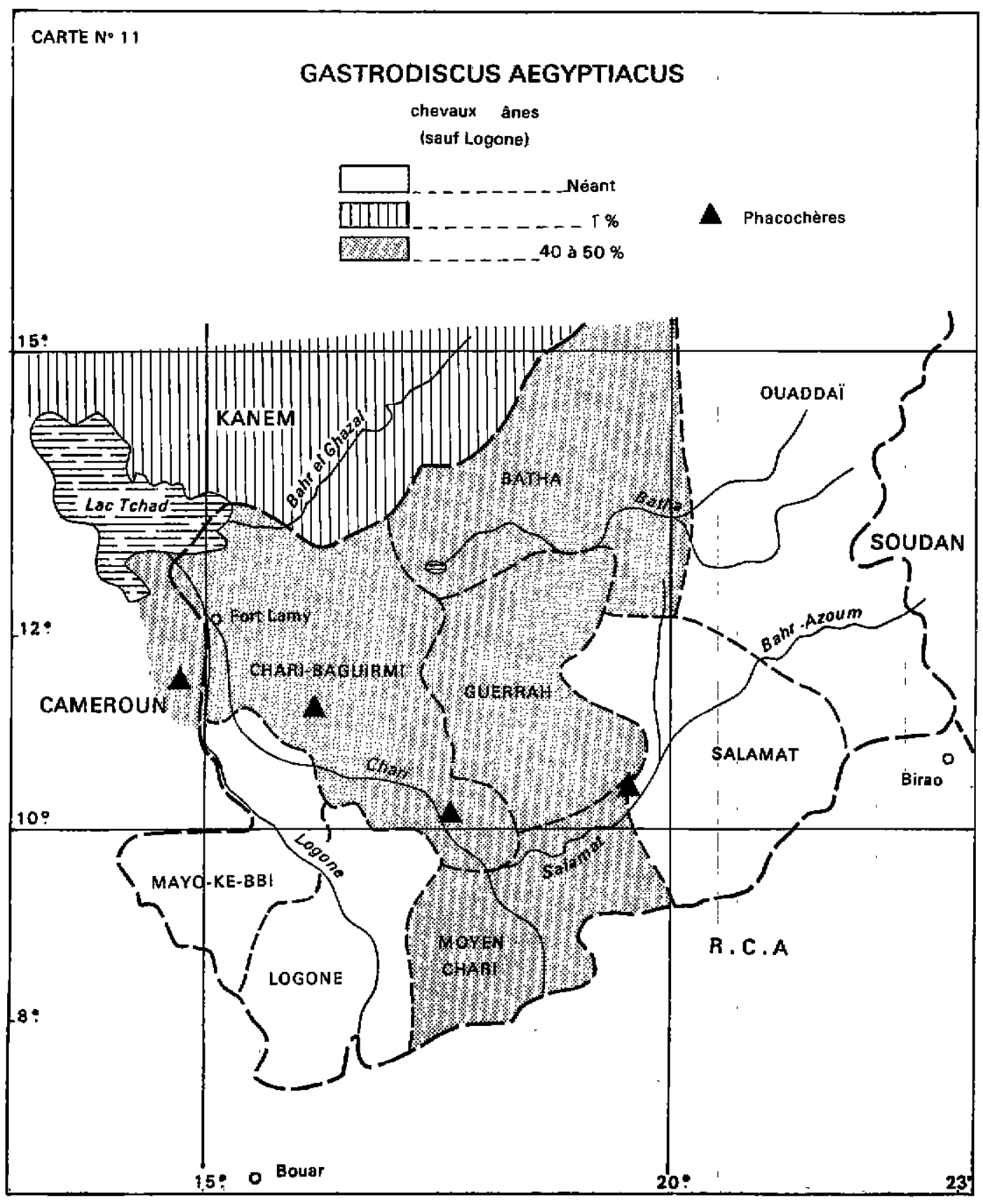




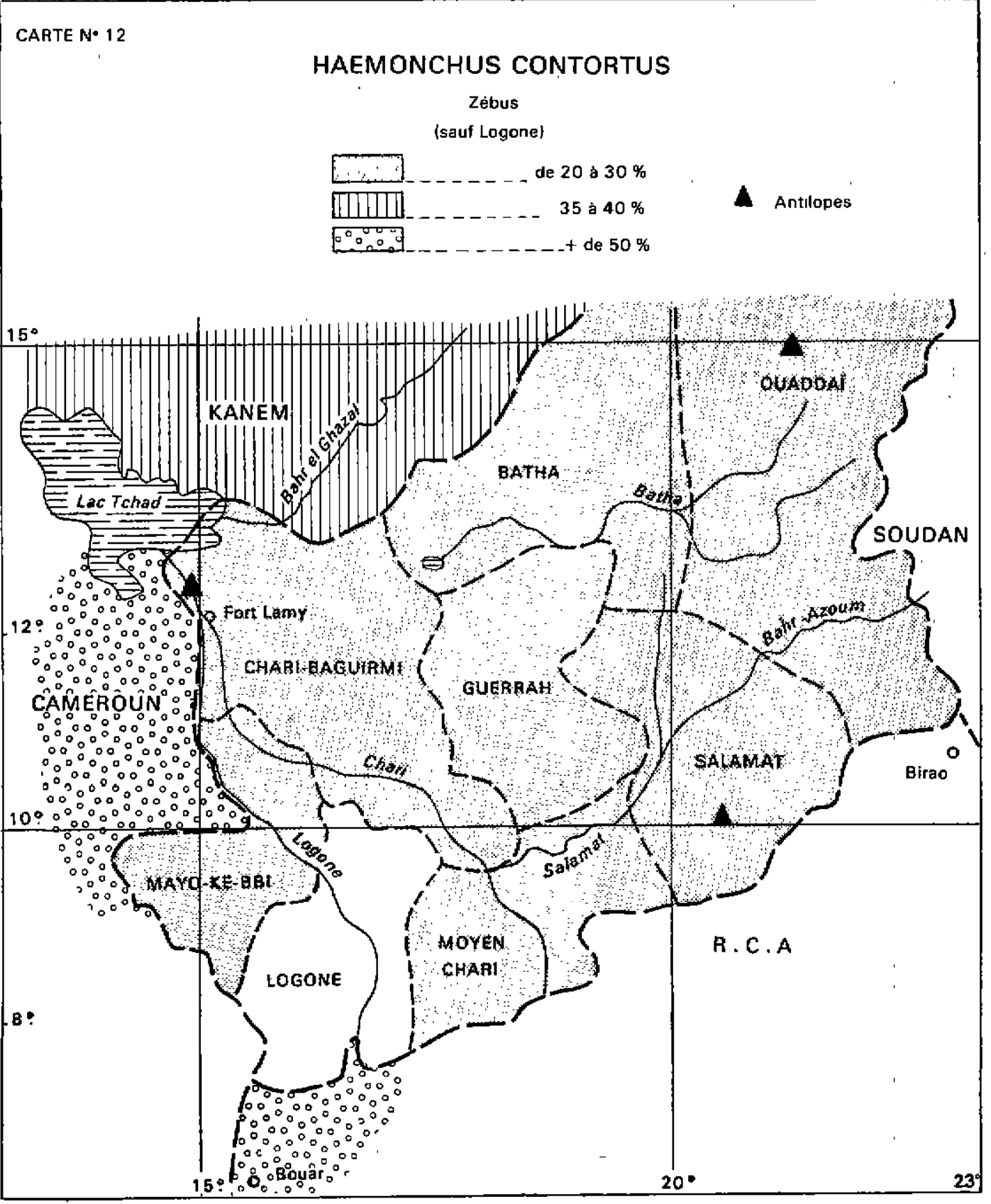


compte de la présence d'Artiodactyles sauvages porteurs d'Helminthes non spécifiques.

Lo solution la plus satisfaisante serait, bien entendu, de multiplier la pratique du Ranching pour les animaux domestiques ef de cantonner. le plus possible, les ruminants squvages dans d'immenses réserves où le bétail d'élevage ne serait plus admis.

\section{REMERCIEMENTS}

Nous remercions Messieurs les Docteurs BRODARD, LATOUR, YVORE, ITARD, COUPET, GRUVEL, et Monsieur ROLLAND d'avoir bien voulu confier à la section d'Helminthologie du Laboratoire de Farcha (Tchad) la détermination de parasites recueillis au cours de diverses tournées en brousse.

\section{LISTE SYSTÉMATIQUE DES HOTES ET DE LEURS HELMINTHES}

\section{1. - Phacochoerus aethiopicus (PALLAS).}

Gastrodiscus aegyptiacus (COBBOLD, 1876). Moniezia mettami (BAYLIS, 1934). Ascoris phocochoer (GEDOELST, 1916). Murshidia pugnicaudato (LEIPER, 1909). Daubneyia m'wanzee (DAUBNEY, 1924). Daubneyia olds (GOODEY, 1924).

Daubneyia roubaudi (DAUBNEY, 1926). Physocepholus sexalotus (MOLIN, 1860). Artionema congolensis (RAILLIET et HENRY, 1911).

2. - Syncerus caffer aequinoxialis (BLYTH).

Fasciola gigantica (COBBOLD, 1855).

Cotylophoron cotylophorum (FISCHOEDER, 1901).

Cormyerius spotiosus (BRANDES, 1898).

Carmyerius endopopillatus (DOLLFUS, 1962).

Cysticercus bovis (COBBOLD, 1866).

Hoemonchus sp.

Artionemo labiato-papilloso (PERRONCITO, 1882.) N. Comb.

3. - Trogelophus scriptus (PALLAS).

Crossotaenio baerı (MAHON, 1954).

\section{4. - Strepsiceros strepsiceros (PALLAS).}

Haemonchus veglial (LE ROUX, 1929).
5. - Alcelaphus lelwel (HEUGLIN).

Colicophoron colicophorum (FISCHOEDER, 1901).

Cotylophoron cotylophorum (FISCHOEDER, 1901).

Carmyerius spatiosus (BRANDES, 1898).

Avifellina sandground (WOODLAND, 1935).

Cysticercus dromedarii (PELLEGRINI, 1954.

Agriostomum cursoni (MONNING, 1932).

Longistrongylus meyeri (LE ROUX, 1929).

Haemonchus contortus (RUDOLPHI, 1803 : COBBOLD, 1898).

Artionemo poultoni (TWAITE, 1927) $\mathrm{N}$. Comb.

Pygorginema ofricono(CHABAUD et ROUSSELOT, 1956).

\section{6. - Damaliscus korrigum (OGILBY).}

Poramphistomum microbothrium (FISCHOEDER, 1901).

Cormyerius spatiosus (BRANDES, 1898). Cormyerius exoporus (MAPLESTONE, 1923). Carmyerius porvipapillatus (GRÉTILLAT. 1926).

Avitellina sandground (WOODLAND, 1935).

Cysticercus dromedorn (PELLEGRINI, 1945). Agriostomum cursoni (MONNING, 1932). Artionemo poultoni (TWAITE, 1927) $\mathrm{N}$. Comb.

Artionemio bicoronato (VON LINSTOW, 1901) N. Comb.

7. - Oryx algazel (OKEM).

Avitellina woodlandi (BHALERAO, 1936).

8. - Hippotragus equinus (DESMARETST).

Paromphistomum microbothrium (FISCHOEDER, 1901).

'Stephanopharynx compactus (FISCHOEDER, 1901).

Cormyerius spotiosus (BRANDES, 1898). Schistosoma boviș (SONSINO, 1876).

Moniezıa monardı. (FURHMANN, 1931).

Avitellino sondground (WOODLAND. 1935).

Stilesio hepotica (WOLFFHUGEL, 1903). Stilesia globipunctato (RIVOLTA, 1874). Cysticercus dromedorii (PELLEGRINI, 1945). Longistrongylus meyer (LE ROUX, 1929). 
Kobusınema schrenki (ORTLEPP, 1939); ORTLEPP 1936 N. Comb.

Bunosfomum dentofum (MONNING, 1931).

Parabronema skjabini (RASOVSKA, 1924).

Artionema hornby' (BOULENGER, 1921) N. Comb.

Buckleyuris globulosa (VON LINSTOW. 1901).

Linguatula nuttalı (SAMBON, 1922).

9. - Addax nosomaculalus (BLAINVILLE).

Avitellina woodland (BHALERAO, 1936).

J0. - Redunca redunca nigeriensis (BLAINE).

Paramphistomum microbothrium (FISCHOEDER, 1901).

Stephonopharynx compoctus (FISCHOEDER, 1901).

Cormyerius spotiosus (BRANDES, 1898).

Stilesio hepatico (WOLFFHUGEL, 1903).

Cysticercus dromedorn (PELLEGRINI, 1945).

Artionemo bicoronato (VON LINSTOW, 1901) N. Comb.

11. - Redunca redunca (PALLAS).

Artionemo bicoronata (VON LINSTIOW, 1901), N. Comb.

12. - Adenota kob (ERXLEBEN).

Fasciola gigantica (COBBOLD, 1855).

Poromphistomum microbothrium (FISCHOEDER, 1901).

Colicophoron calicophorum (FISCHOEDER, 1901).

Stephonopharynx compoctus (FISCHOEDER, 1901).

Cormyerius spatiosus (BRANDES, 1898).

Carmyerius popillatus (GRÉTILLAT, 1962).

Carmyerius porvipapillatus (GRETILLAT, 1962).

Schistosoma bovis (SONSINO. 1876).

Avifellina sandgroundi (WOODLAND. 1935).

Cysticercus bovis (COBBBOLD, 1866).

13. - Kobus defassa (RUPPEL).

Paramphistomum microbothrium (FISCHOEDER, 1901).

Stephonophorynx compactus (FISCHOEDER, 1901).

Carmyerius spatiosus (BRANDES, 1898).

Carmyerıus papillatus (GRÉTILLAT, 1962).
Carmyerius parvipapillatus (GRÉTILLAT, 1962).

Schistosomo bovis (SONSINO, 1876).

Moniezio monardi (FURHMANN, 1931).

Stilesio hepotica (WOLFFHUGEL, 1903).

Cysticercus dromedarii (PELLEGRINI, 1945).

Agriostomum cursoni (MÖNNIG, 1932).

Bunostomum dentatum (MÖNNIG, 1931).

Longistrongylus meyerı (LE ROUX, 1929).

Haemonchus sp.

Artionema bicoronota (VON LINSTOW, 1901) N. Comb.

Buckleyuris globulosa (VON LINSTOW, 1901).

Cooperia punctata (VON LINSTOW, 1906 ; RANSOM, 1907).

14. - Gazella dorcas dorcas (LINNÉ).

Haemonchus contortus (RUDOLPHI, 1803 ; COBBOLD, 1898).

Gazellofilaria tanganyikae (YEH, 1955).

Artionemo scolprum (VON LINSTOW, 1908) N. Comb.

Buckleyuris globuloso (VON LINSTOW, 1901).

15. - Gazella rufifrons (GRAY).

Poramphistomum microbothrium (FISCHOEDER, 1901).

Cysticercus bovis (COBBOLD, 1866).

Cysticercus dromedarii (PELLEGRINI, 1945).

Oesophagostomum columbionum (CURTICE, 1890, RAILLER et HENRY, 1913).

Hoemonchus contortus (RUDOLPHI, 1803 ; COBBOLD, 1898),

Cooperio sp.

Artionema scolprum (VON LINSTOW, 1908) N. Comb.

Artionemo bicoronota (VON LINSTOW, 1901) N. Comb.

Buckleyuris globulosa (VON LINSTOW, 1901).

16. - Ourebia ourebi (ZIMMERMAN).

Artionema scalprum (VON LINSTOW. 1908) N. Comb.

17. - Ourebia ourebi dorcas (Schw.).

Arfionema bicoronata (VON LINSTOW, 1901) N. Comb.

18. - Ourebra ourebi splendida (Schw.).

Artionema bicoronata (VON LINSTOW, 1901) N. Comb. 


\section{SUMMARY}

Helminths of certain wild artiodactyla belonging to the bovidae and the suidae in the Republics of Chad and Central Africa. Are these mammals reservoirs of parasites for domestic animals living in contact with them ?

In CHAD and R. C. A., the authors have carried on autopsies of approximately a hundred wild Bovidae and Suidae, belonging to the following species:

Phacochoerus aethiopicus (10), Syncerus caffer aequinoxialis (3), Tragelophus scriptus (1), Strepsiceros strepsiceros (1). Alcelaphus lelwel (11). Damaliscus korrigum (7), Oryx algazel (1), Hippotragus equinus (9), Addax nasomoculatus (1), Redunco redunca nigeriensis (2), Redunca redunca (1). Adenota kob (6), Kobus defassa ( $\theta$ ), Gazella dorcas dorcos (22), Gazella rufifrons (14), Ourebia ourebi (1), Ourebia ourebi dorcos (1), Ourebia ourebi splendido (1).

A tolal of 47 species of Helminths have been discovered of which 26 were host-specific : Carmyerius exoporus, Cormyerius endopopillatus, Moniezio monordi, Avitellina sandgroundi, Maniezia mettomi. Crassotoenio boeri, Longistrongylus meyeri, Longistrongyius aibifrontis, Kobusinema schrenki. Hoemonchus veglia, Parabronema skrjabin!, Ascaris phocochoeri, Agriostomum cursoni, Bunostomum dentatum, Oesophagostomum m'wanzee, Oesophagostomum oid, Oesophagostomum roubaudi, Pygarginema africana, Murshidia pugnicaudota, Artionemo congolensis, Artionema scalprum, Artionema hornbyi, Artionema bicoronata, Artionema poultoni, Gazellofilaria tanganyikae, Linguatula nuftali.

The remaining twenty one could infect wild and domesticated Artiodactyla

a) Eight of them appear to be proportionately more common in the Buffalo and the Antelope than in the Zebu, sheep and goat, in which the infestation is small. The transmission from one animal group to another is due to the frequenting of the same pastures, ce, and their migration according to the seasons. These are : Calicophoron colicophorum, Cotylophoran cotylophorum, Stephanophorynx compactus, Carmyerius spatiosus, Cormyerius papillatus, Carmyerius parvipapillatus, Stilesia hepatica and Cysticercus dromedarii.

b) The last thırteen species are very common, being widely dispersed and well establıshed in the country. These are rather parasites of domestic ruminants swine and Equidne, which have inspected wild rumınants under the conditions described above : Poramphistomum micrabothriumi. Hoemonchus contortus, Schistosoma bovis, Fasciola gigantica, Cysticercus bovis, Stilesia globipunctata, Oesophagostomum columbianum, Artionemo labiato-papillosa, Buckieyuris globuloso, Cooperia punctato, Avitellina woodiandi, Gastrodiscus aegyptiocus and Physocephalus sexalatus.

In CHAD and R. C. A, the wild Artiodactyla do not appear, at present, to be a danger to the Bovines, Ovines, Caprines and Camelidae with which they are in contact, since nearly three quarters of the Helminths, discovered are either strictly host-specific or little found in the domestic ruminants. However, regarding the widely dispersed parasites, their importance should not be underest mated (for exemple Haemonchus contortus of Gazelia dor cos and Gazella rufifron in the semi-desert regions).

The authors would also point out that the parasitic fauna of the wild Bovidae and Suidae of the basin of the CHARI-LOGONE does not differ greatly from those of the basins of the NILE or the CONGO or those of the countries of South and East AFRICA.

The pathogenic action of the Heiminths and the parasitic associations are also thought to be similar.

Twelve maps and ample references are provided with this paper. 


\section{RESUMEN}

Los helminlos de algunos artiodactilos salvajes perteneciendo a las familias bovinas y porcinas. l Son estos mamiferos, en la Republica del Tchad yen R. C. A., depositos de parásitos para los animales domésticos viviendo en contacto con ellos?

Los autores, en el Tchad en R. C. A., procedieron a la autopsia de una centeno de bovinos y porcinos salvajes perteneciendo a las especies siguientes :

Phacochoerus aethropicus (10), Syncerus caffer aequinoxiolis (3), Tragelophus scriptus (1), Strepsiceros strepsiceros (1), Alcelaphus lelwel (11), Damaliscus korrigum (7), Oryx algazel (1). Hippotragus equinus (9), Addax nosomoculofus (1), Redunca redunca nigersensıs (2), Redunco redunco (1), Adenola kob (6), Kobus defasso (8), Gozello dorcas dorcas (22), Gazella rufifrons (14), Ourebio ourebi (1), Ourebia ourebi dorcas (1), Ourebio ourebi splendido (1).

En total, se encontroron 47 especies de helminios entre los cuales 26 específicos : Carmyerius exaporus, Carmyerius endopopillotus, Moniezio monardi, Avitellino sandgroundi, Crossotaenia boeri, Longistrongylus meyeri, Longistrongylus albıfrontıs, Kobusınema schrenki, Haemonchus veglıai, Parabronema skjobın, Ascaris phocochoerı, Agriostomum curson!, Bunostomum dentolum, Oesophagostomum m'wanzee, Oesophogostmum oldi, Oesophogostornum rouboudi, Pygorginemo africana, Moniezia mettami, Murshidia pugnicoudata, Artionema congolensi, Artionema scalprum, Artionema hornbyi, Artionema bicoronoto, Artionema poultoni, Gazellofilario tongonyikae, Linguotulo nuttali.

Los otros 21 son comunes a los Artiodactilos salvajes y a los doméśicos. Ocho de entre ellos son mós bien parásitos de búfalos a de antílopes que atacan los cebús, la oveja y la cabra, por el medio de la transumancia y mezclas de pablacianes animales : Calicophoron calicophorum, Coiylophoron cotylophorum, Stephanopharynx compactus, Carmyerius spatiosus, Carmyerius papillatus, Carmyerius parvipopillatus, Stilesia hepatica y Cysticercus dromedarii.

Las ultimas trece especies son muy frecuentes, ampliamente dispersadas y fuertemente implantadas en el pais : Paromphistomum microbothrium, Hoemonchus contortus, Schistosoma bovis, Fasciola gigantica, Cysticercus bovis, Stilesia globipunctata, Oesophagostomum columbianum, Artionemo labiato-popilloso, de Buckleyuris globuloso, Coaperia punctata, Avilelinno woodlandi, Gostrodiscus aegyptiacus Physocephalus sexalatus.

En el Tchady en R. C. A., los Artiodaclylos salvajes no parecen, hasta ahora, representar un pelıgro seguro para los bovinos, ovinos, cabrunos o camellos que viven en su proxımidad Sin embargo. en cierlos casos, en suma limitados, no debe ser menospreciado su papel.

Los auiores senalan, además, que la fauna parasitaria de los bovinos y porcinos salvajes de la cuenca del rio Chari-Logone no difiere fundamentalmente de la de las cuencas del Nilo o del Congo y de la de los paises de Africa del Este o de Africa del Sur.

La acción patógena de esios helmintos y la importancia de las asociaciones parasitarias son también examinadas.

12 mapas de repartición geográfica y una importante bibliografia acompanan el presente documenio. 


\section{BIBLIOGRAPHIE}

1. ANTIPIN (D.N.), ERSHOW (V.S.), ZOLOTAREV (N. A.) et SALYAEV (V. A.). Parasitology and parasitif diseases of Livestock, 1956, Moscow (Traduction 1960, Israel program. Sci. Trans), 464, 308 fig.

2. ALMEIDA (L. J.). - Revisao do genero Haemonchus Cobb, 1898. Mem. Inst. Osw. Cruz, 1935, $30: 57-114$.

3. ARMFIELD(J.M.). - Parasites of the Grant's Gazelle. Vet. Rec. 1922, 2, 263.

4. - BAER (J. G.). - Résultats zoologiques du voyage du Dr P. A. Chappuis au Nil supérieur III: Helminthes. Rev. Sulsse zool., 1923, 30 (13) : 344-51, 11 fig.

5. BAER (J. G.). - Contribution d̀ la faune helminthologique sud-africaine. Ann. Parasit. hum. comp., 1924, 2 (3) : 239-47.

6. BAER (J,G.). - Contribution to the Helminth fauna of South Africa. Mamalian Cestodes: $11^{\text {th }}$ and 12 th Rep. Dir. Vet. Educ. Res., Union South Afr., Prétoria, 1926: 62-135, 40 fig.

7. BAER (J.G.), - Monographie des Cestodes de la famille des Anoplocephalidae. Bull. Biol. France et Belgrque, suppl, 10, $1927: 241$, 43 fig.

8. BAER (J. G.) et FAIN (A.). - Cestodes Exploration du parc national de l'Upembal. Mission C. F. de Witte. Inst. Parcs nat. Congo Belge, Bruxelles 1955 (36) : 1-37, 12 fig.

9. BAER (J.G.) - Exploration des parcs nationaux du Congo Belge - Mission J. G. Baer - Helminthes parasites. Inst. Parcs Nat. Congo Bel., 1959 (1) 163, 92 fig. 7 pl.

10. BATTELI (C.). - II. Cys. dromadarri, Pellegrini 1945 in Eritrea. Bull. Soc. Hal. Med. lg. Trop. Asmora, 1947, 9 (3) : 289-94.

11. BAYLIS (H. A.). - Some considerations on the host distribution of parasitic Nematodes. J. Linn. Soc. (Zool.), 1923, 36: 12-43.

12. BAYLIS (H. A.) ef DAUBNEY (R.). - A synopsis of the families and genera of Nematoda. London, 1926.

13. BAYLIS $(H$. A.). - On two adult Cestodes from wild swine. Ann. Mag. nat. Hist. 1927, 19 (9) : 417-25.
14. BAYLIS ( $H$. A.). - Three notes on parasitic Nematodes. Ann. Mog. nat. Hist. (ser. 10), 1932, $10: 497-502,3$ fig.

15. BAYLIS $(H$. A.). - Note on four Cestodes. Ann. Mag. nat. Hist. 1934, 14 : 587-93.

16. BAYLIS (H. A.). - Some parasitic worms from the Cameroons. Ann. Mog. nat. (a) Hist. (ser. 10), 1936, 71 : 157-272, 11 fig.

17. BAYLIS (H. A.). - Fauna of British India - Nematoda I. London, Taylor et Francis. 1936: 408 .

18. BAYLIS (H. A.). - Records of some parasitic worms from the Belgian Congo. Ann. Mog. nat. Hist. (ser. 11) 1939, $3: 625-9$.

19. BEAUCHAMP ( $P$, de). - Sur quelques Helminthes provenant du Congo Belge. 1914.

20. BHALERAO (G. D.). - On some representatives of Cestode genus Avitellino from India. 1. Helminth, 1936, 14 (3) : 141-62, 21 fig.

21. BOULENGER (C. L.). - On some Filariid parasites of cattle and other ruminants. Parasitology 1921, 12 (4) : 341-9, 17 fig.

22. BOULENGER (C. L.). - Report on a collection of parasitic Nematodes, mainly for Egypt Part V, Filaroidea Parasitology, 1928, $20: 32-55$.

23. BRANDES (G.). - Die gattung Gastrothylax. Noturforsh. Gezellsch. Z: Halle., 1898, 21 : 195-225, 8 pl., 16 fig.

24. CAEIRO (V. M. P.). - Acerca de alguns Paramphistóminae nâo assinalados em territorios portugueses. Rev. Ciên. Vet. (Lisbonne) 1961, 56 (377) : 68-106.

25. CALL (C.). - II. Cysticercus dromedarii Pellegrini in un' Antilope Eritrea. Boll. Soc. it. Med. Ig. Trop. Asmara, 1949, 9 (3) : 300-2.

26. CHABAUD (A. G.) et ROUSSELOT (R.) (d). - Sur quelques filaires d'Afrique équatoriale. Ann. Parasit. hum. comp., 1956, 31 (1-2) : 53-98, 23 fig.

27. CHABAUD (A. G.) et ROUSSELOT (R.) (b). --Pygarginemma africana n. sp. (Nematoda, Ascaropsinae) parasite d'un Céphalophe africain. Ann. Parasit. hum. comp., 1956, 31 (3) : 248-54, 3 fig. 
28. CHABAUD (A. G.). - Revue critique des Nématodes du genre Quinonia Lane 1914 et du genre Murshidia, Lane 1914. Ann. Parosit. hum. comp., 1957, 32 (1-2) : 98-131, 8 fig.

29. COCEANI (C.). - Frequenza del Cystlcercus bovis e die $C$. dromedarii tra gli zebu eritrei. Boll. Soc. It. Med. Ig. Trop. Asmara, 1949, 9 (3) : 295-99.

30. CONFÉRENCE NAIROBI. - Fauna of british eastern and central africa. Conférence Nalrobı, 1948 (1) : 74.

31. DAUBNEY (R.). - A note on two species of the genus Murshidia (Nematoda, Strongyloidea) parasitic in a wart-hog. Ann. Mag. nat. Hist. (ser. 9), 1923, II : 256-63, 10 fig.

32. DAUBNEY (R.). - Description of a new Nematode, Esophagostomum m'wanzee, from the wart-hog. Ann. Mag. not. Hist., $1924,13(9): 542-6$.

33. DAUBNEY (R.). - Esophagostome from the wart-hog. Ann. Mag. nat. Hist. (ser. 9), 1926, $17: 11-17$.

34. DAWES (B.). - On a collection of Paramphistomidae from Malaya,' with revision of the genera Paramphistomum Fischoeder, 1901, and Gastrothylax Poirier 1883. Parasitology 1936, 28 (3) : 330-54 fig. IA-7 C.

35. DAWES (B.). - The trematoda. Cambridge. $1946: 644,81$ fig.

36. DINNIK (J. A.), DINNIK (N. M.). - The life-cycle of Paramphistomum microbothrium Fischoeder, 1901 (Trematoda : Paramphistomidae). Parasitology, 1954, 44 : 285-99.

37. DINNIK (J. A.) et DINNIK (N. M.). - Development of Carmyerius exoporus Maplestone (Trematode : Gastrothylacidae) in a snail host. Parasitology 1960, 50(3-4) : 469-80, 8 fig.

38. DINNIK (J. A.). - Paramphistomum phillerouxi Sp. Nov. (Trematoda : Paramphistomidae) and its development in Bulinus forskalii. J. Helminth, 1961. (1-2), 69-90.

39. DINNIK (J. A.), - Moniezia monardi Furhmann and Avitellina buechneri Sp. Nov. from Adenota Kob thomasi. J. Helminth $19630,37(3): 169-78,5$ fig.
39'. DINNIK (J. A.), WALKER (J. B.), BAR$\operatorname{NETT}$ (S. F.) and BROCKLESBY (D. W.). Some parasites obtained from game animals in Western Uganda. Bull. Epiz, dis. Afr., 1963 b, II (1): 37-44.

40. DOLLFUS (R. P.). - Helminthal Trematoda et Acamthocephala. Contribution à la Faune du Cameroun, 2e Fasc. Faune des colonies françaises. Paris, 1929 : 73-114, 23 fig.

41. DOLLFUS (R. P.). - Mission scharienne Augıèras-Draper. Trématodes des mammifères, olseaux et poissons. Bull.' Mus. Hist. not. (ser. 2), 1932, 4 (5) : 555-63.

42. DOLLFUS (R. P.). - Trématodes récoltés au Congo Belge par le Professeur P. Brien (mal-août 1937). Ann. Mus. r. Congo Belge, C. Zoologie (ser. 5), 1950, I1 :1-136.

43. DOLLFUS (R. P.). - Variations intraspécifiques chez un Carmyerius (Trématoda ; Gastrothylacidae) parasite du buffle du Congo Belge. Ann. Porosit. hum. comp.1962, $37(1-2)$ : 108-20, $13 \mathrm{fig}$.

44. DOLLFUS(R.P.). - Hôtes ef lieux de récolte de quelques Trématodes digénétiques de vertébrés de la collection du Musée royal de I'Afrique centrale. Rev. Zool. Bot. Afr., 1963, 68 (3-4) : 323-57, 7 fig.

45. EUZEBY (J.) (a). - Les maladies vermineuses des animaux domestiques et leurs incidences sur la Pathologie humaine. 1-1 Parıs, Vigot Frères, $1961: 450,1,64$ fig.

46. EUZEBY (J.) (b). - Ibidem, 1963 (1-2) : 844, 268 fig.

47. EZZAT (A. E.). - - Helminth parasites of some ungulates from the Giza zoological gardens, Egypt. Bull. Minist. Agric. Egypte, 1945, 241 : 104, 119 fig.

48. FAIN (A.). - Les Pentastomidés de l'Afrique centrale. Ann. Mus. r. Afr. cent. (Belgique), 1961 (92) : 115, 89 fig., 6 pl.

49. FISCHOEDER(F.).-Die Paramphistomiden der sâugethiere. Zoolog. Anzeıger, 1901, 24 (646) : 367-75.

50. FISCHOEDER(F.).-Die Paramphistomiden der saugethiere. Dissertation Universitał Königsberg, 1902 : 1-59, 4 fig. 
51. FISCHOEDER(F.). - Die Paramphistomiden der säugethiere. Zool. Johrb. System 1903, $17(5-7)$ : 485-660, 104 fig.

52. FUKUI (T.). - Studies on Japanese Amphistomatous parasites, with a revision of the group. Jop. J. Zool., 1929 (3) : 219-351, 45 fig.

53. FUHRMANN (O.). - Die Cestoden der Vôgel des Weissen Nils. Res. Swedish Zool. Exp. Egypt and White Nil, 1901 (27) : 55-53 fig.

54. FUHRMANN (O.), - Dritte klasse des Cladus Plathelminthes, Cestoidea : Cyclophyllidea. In handbuch der zoologie... gegründet von dr Willy Kukenthal... herausgegeben von $\mathrm{Dr}$ Thilo. Krunbach. Berlin und Leipzig, 1931, 2 : 141-416.

55. FUHRMANN (O.). - Deux nouveaux Cestodes d'Angola. Bull. Soc. Neuchâtel, Sci. nat. 1933, 58 : 97-106, 7 fig.

56. FUHRMANN (O.) et BAER (J. G.). - Mission Sagan-Omo (Ethiopie méridionale) 1939 Cestodes. Bull. Soc. Neuchôtel, Sci, nat., 1943, 68 : $113-40,22$ fig.

57. GeBAUER (O.). - Zur Kenntnis der Parasitenfauna der Gemse. Z. Parasitenk., 1932, $4: 147-220$.

58. GEBAUER (O.). - Ein neur Wiederkaür peitschenwurm (Trichuris gazellae) n. sp. aus der Dama gazelle. Z. Parasitenk, 1933, $16: 321-25$.

59. - GEDOELST (L.). - Synopsis de Parasitologie de l'homme ef des animaux domestiques. Bruxelles, 1911.

60. GEDOELST (L.). - Notes sur la faune parasitaire du Congo Belge. Rev. Zool. Afr. 1916, 5 (1) : 1-90, 20 fig.

61. GOLVAN (Y.), CHABAUD (A.) et GRETILLAT (S.). - Carmyerius dollfusi n. sp. (Trématoda : Gastrothylacidae) parasite des bovidés à Madagascar. Ann. Parasit. hum. comp., 1957, 32 (1-2) : 56-70, 9 fig.

62. GOODEY (T.). - Some new members of the genus Fsophagostomum from the Roan Antelope and the wart-hog. I. Helminth, 1924,2 (3) : 135-48.
63. GOUGH (L. W.). - A monograph of the Tapeworms of the subfamily Avitellinae being a revision of the genus Stilesia and an account of the histology of Avitellina centripunctato (Riv.) Quart. J. Microscop. Sci. 1911, $56: 316-85,42$ fig.

64. GRABER (M.) et RECEVEUR (P.). - Parasifisme interne du mouton en zone sahélienne. Esophagostomose nodulaire en particulier. Rev. Elev. Méd. vét. Pays trop., 1956, 9 (2) : 5-20.

65. GRABER (M.). - La Cysticercose bovine ; son importance dans les zones sahéliennes d'élevage de la République du Tchad. Rev. Elev. Méd. vét. Pàs trop., 1959, 12 (2) : 121-43.

66. GRETILLAT (S.) (a). - Amphistomes (Trematoda) des Ruminants domestiques de la République du Tchad ; description d'un Gastrothylacidae nouveau Carmyerius graberi n. sp. Ann. Parasit. hum. comp., 1960, 35 (4) : 509-27, 9 fig.

67. GRETILLAT (S.) (b). '- Structure anatomique du diverticule pharyngien dans l'espèce Stephanopharyns compactus. C. R. Acad. Sci., 1960; 250: 4064-66.

68. GRETILLAT (5.). - Carmyerius papillatus n. sp. ef Carmyerius parvipapillatus n. sp. (Trematoda : Gastrothylacidae) parasites des réservoirs gastriques de l'Antilope Kobus defassa (Rüppl). Ann. Parasit. hum. comp., 1962, 37 (1-2) : 131-39, 15 fig.

69. GROBBELAAR (C. S.). - On south african Paramphistomidae. Trans. R. Soc. S. Afr., 1922, $10: 181,90$.

70. HALLORAN (P.). - A bibliography of references to diseases of wild animals and birds. Amer. J. vet. Res., 1955, 16 (61) : 2-465.

71.' HEYMONS (R.) ef VITZHUM (H. G.).Beitrage zur'systematik der Pentastomiden. Parasitenk., 1935, 7 (1) : 103, 36 fig.

72. HUDSON (J.R. . . - A list of Cestodes known fo occur in east african animals, birds and reptiles. J. E: Africo Uganda not. Hist. Soc., 1934, 49 i: 205-17.

73. INNES (J. A.). - Gastrothylax bubalis $n$. sp., with a few notes on the genus gastrothylax (Poirier). Porasitology, 1912, $5: 217$. 25. 
74. JOYEUX (C. K.). - Liste de quelques Helminthes récoltés dans les colonies portugaises d'Afrique. Ann. Porasit. hum. comp., 1924, 2 (3) : 232-35.

75. JOYEUX (C.), GENDRE (E.) et BAER (J.G.). - Recherches sur les Helminthes d'A.O.F. Coll. Soc. Path. Exot. Monographie II Paris, Mason, 1928 : 120, 52 fig.

76. JOYEUX (C.) ef BAER (J. G.). - Faune de France. 30 - Cestodes - Paris' 1936, 610, 560 fig.

77. KREIS (H. A.). - Beitràge zur kenntlis parasitischer Nematoden I Ein never parasitischer nematode aus der hirshziegenantilope, Antilope cervicapra L. : trichuris cervicapra. Verh. Natur. Ges. Bosel., 1935 (142) : 90-105.

78. KREIS (H. A.) (o). - Beitrâge zur kenntnis parasitischer Nematoden. 7 Parasitische Nematoden der schweizerischen wissenschaftlichen. Expedition nach Angola In jahre 1932. Zbl. Bokt., 1938 (142) : 90-105.

79. KREIS (H. A.) (b). - Beitràge zur Kenntnis parasitlscher Nematoden 8 Neue Parasitische Nematoden aus dem Naturhisorischen Museum Basel. Zbl. Bakt. Porosit., 1938, I42 (1) : 329-52, 12 fig.

80. LANE (C.). - Some strongylata. Porasitology, 1923, $14:$ 348-64.

81. LAPAGE (G.). - Veterinary parasitology. Edinburgh, 1956: 964, 494 fig.

82. LEINATI (L.), MARAZZA (V.), GRIMALDI (E.) et PERSIANI (G.). - Le elminliasi dell' vomo da alimenti di origine animale. Clinica vet. (Milano) 1963 (86) : 173-217, 242-57, 356-405, 65 fig.

83. LEIPER (R. T.). - An account of some helminths contained in $\mathrm{Dr} C$. N. Wenzyon's collection from the Sudan. III d Rep. Wellc. Res. Laborat. Khortoum, 1908 : 187 99, 12 fig.

84. LEIPER (R. T.). -- Wissensch ergebuisse der Schewedischen zoologischen expedition nach dem Kilimandjaro, dem Meru etc... Stockholm 22 - Vermes, 1909 : 23-36, 9 fig.

85. LEIPER (R. T.). - The entozod of the Hippopotamus. Proc. Zool. Soc. London, 1910, 19 (ser. 4) : 233-51.
86. LEIPER (R, T.). - Observation on certain Helminths of man. Trans. R. Soc. trop. Méd. Hyg., 1913, $6: 265$.

87. LE ROUX (P.L.) (a). - A preliminary report on three new members of the genus Haemonchus Cobb. 1898 from Antelopes in South Africa. 15 th Rep. Dir. Vet. Serv. Anim. Ind. Union S. Afr. 1929 : 451-62, 22 fig.

88. LE ROUX (P. L.) (b). - On a hookworm (Agriostomum gorgonis n sp.) from the blue wildbeast (Gorgon taurinus) in the Transvaal. 15 th. Rep. Dir. Vet. Serv. Anim. Ind. Union S. Afr., 1929: 481-91, 13 fig.

89. LE ROUX (P. L.). - On Longistrongylus meyeri gen. and sp. nov. a trichostrongyl parasitizing the red Hartebeest Bubalus caama. J. Helminth, 1931, $9: 141-6$.

90. LE ROUX (P. L.). - A preliminary note on Bilharzia margrebowie, a new parasite of ruminants and possibly of man in Northern Rhodesia. J. Helminth, 1933, II (1) : 57-62.

91. LE ROUX (P. L.). - On the division of the genus Esophagostomum Molin', 1861, into subgenera and the creation 'of an new genus for the Esophagostomes of the wart-hog. J. Helminth, 1940, 18 (1) : 1-20, $23 \mathrm{fig}$.

92. VON LINSTOW (O.). - Nematoden anus der berliner zoologischen sammlung. Mitt. zool. Mus. Berl., 1899, I : 3-28.

93. VON LINSTOW (O.). - Helminths von den ufern des Nyassa-sees, ein Beitrag zur Helminthen-Fauna von Suid-Afrika. Jena. $Z$. Noturw., 1901, 35 : 409-28.

94. VON LINSTOW (O.). - Beocachtungen an Nematoden und Cestoden. Arch. Naturgesch, 1904 (70) : 297-309.

95. VON LINSTOW (O.). - Nematoden aus dem Kôniglichen Zoologischen Museum in Berlin. Mitf. zool. Mus. Berl., 1907, 3 : 251-9.

96. VON LINSTOW (O.). - Helminths Nematoden und Acanthocephalen. Denkschr. Med. naturw. Ges. Jena, 1908, 13 : 19-28

97. LOPEZ-NEYRA (C. R.). - Helminthos de los vertebrados ibéricos. 1947, I : 407. 
98. LOOSS (A.) - Recherches sur la faune parasitaire de l'Egypte. Mem. Inst. Egypte, Le Coire, 1896, 3 : 1-252, 193 fig.

99. MAHON (J.). - Tapeworms from the Belgian Congo. Ann. Mus. r. Congo Belge $C$. Zool. 1954 (ser. 1) (2) : 141-261, 74 fig.

100. MALBRANT (R.), - Faune du Centre africain français. Parıs, 1952 : 616, 129 fig.

101. MALEK (E.). - Chek list of Helminth parasiles of domesticated animals in Sudan. Ind. vet. J., 1959, 36 (6) 281-8.

102. MAPLESTONE (P. A.). - Revision of the amphistomata of mammals. Ann. trop. Med. Porasit., 1923, 17 (2) : 113-212, 32 fig., 8 pl.

103. MAPLESTONE (P. A.). - Parasitic Nematodes obtained from animals dying in the Calcutta zoological gordens. Rec. Ind. Mus., 1931, $33: 71-171,156 \mathrm{fig}$.

104. MARTINAGLIA(G.). - Rep. of abattoir and livestock market. 1932 (22) S. Afr.

105. MARTINAGLIA (G.).- - Some considerations regarding the health of wild animals in captivity. S. Afr. J. Sci., 1937, 33 : 833-44.

106. Mc. DIARMID (A.). - Maladies des animaux sauvages vivant en liberté. Monograph. F. A.O. Rome, $1964: 127$.

107. MEGITT (F. J.). - Cestodes of mammals. London 1924 : 282.

108. MONNIG (H. O.). - South african parasitic Nematodes. 9 th a. 10 th Rep. Dir. Vet. Educ. Res. Onderstepoort, I, 923, 1, 435-78, 46 fig.

109. MONNIG (H. O.). - Checklist of the worm parasites of domesticated animals in South Africa. 13 th a. 14 th Rep. Dir. vet. Servi. Union S. Afr. 1928, 801-37, 42 fig.

110. MONNIG $(\mathrm{H}, \mathrm{O}$.$) . - Wild antelopes as$ carriers of Nematode parasites of domestic ruminants. Part. I. 17 th Rep. Dir. Vet. Serv. Anım. Ind. Union S. afr., 1931, 1, 233-54, 25 fig.

111. MONNIG $(\mathrm{H} . \mathrm{O}$.) (a). - Wild antelopes as carriers of Nematode parasites of domestic ruminants. Part. II. 18 th. Rep. Dir, Vet. Serv. Anım. Ind. Union S. Afr., 1932, 1, $153-72,27$ fig.
112. MONNIG ( $\mathrm{H} . \mathrm{O}$.) (b). - The genus Agriotomum with a description of A. cursoni n. s. sp. J. S. Afr. Vet. Med. Ass., 1932, 3 (1) : 16-21, 6 fig.

113. MONNIG (H. O.) (c). - New strongylid nematodes of Antelopes (Preliminary notes). J. S. afr. vet, med. Ass., 1932, $3: 171-5$.

114. MONNIG (H. O.) (o), - Wild Antelopes as carriers of Nematode parasites domesic ruminants. Onderstepoort $\mathrm{f}$. vet. Scl. Anim. Ind., 1933, I (1) : 77-92, 27 fig.

115. MONNIG (H. O.) (b). - A new species of Setaria from Antelopes. J. S. Afr. vet, med. Ass., 1933, 4 (1) : 21-3, 4 fig.

116. MONNIG (H. O.). - Cooperia yoshidai n. sp., a Nematode parasite of Reedbuck, Redunca arundinum. Vol.'Jub. Prof. Yoshida, $1939: 291-94$

117. MONNIG (H, O.). - Veterinary Helminthology and Entamology. London, 1950 : 420,275 fig.

118. MOREL(P.). - Les Helminthes des animaux de l'Afrique occidentale. Rev. Elev. Méd. vét. Poys trop., 1959, I2 (2) : 153-74.

119. NAGATY $(H . F$.$) . - An account of the ana-$ tomy of certain Cestodes belonging to the genera Stilesia and Avitellina. Ann. trop. Méd. Parasit., 1929, 23 : 349-80.

120. NASMARK (K. E.). - A revision of the Tre. matode family Paramphistomidae. Inaug. Dissert. Zool. Bidrag. Uppsala, 1937, 16 : 301-566, 104 fig. $13 \mathrm{pl}$.

121. NEVEU-LEMAIRE (M.). - Les Esophagostomes des Phacochères. Ann. Porosit. hum., comp., 1927, 5 (3) : 214-19.

122. NEVEU-LEMAIRE (M.), - Traité d'Helminthologie médicale et vétérinaire. Paris, 1936: 1514, $78 \mathrm{fig}$.

123. ORTLEPP (R. J.). - On some Helminths from the «Nyighiae » Boselaphus tragocamelus (Pall.) with observations on the parasitic larval stages of the stomach worm Ashworthius martinagliai n. sp. Onderstepoort. J. vet. Sci. Anim. Ind. nv, 1935 (1): 43-50, 9 fig.

124. ORTLEPP $(R, J$.$) . - Whipworms from$ South african ruminants. Onderstepoort $\mathrm{J}$. vet. Sci. Anim. Ind., 1937, 9 (1) : 91-100, 7 fig. 
125. ORTLEPP (R. J.). - South african Helminths - Part. VI. Some Helminths chieffly from rodents. Onderstepoort $\mathrm{J}$, vet. Sci. Anım. Husb., 1939, 12 (1) : 75-101, 19 fig.

126. ORTLEPP (R. J.). $-N$ corsing van Suidafrlkaanse Helminte veral met verwysing na die wat in ons wildherkovers voorkom. Tydskr. V. Notuur. Pretoria, 1961, 1 (2) : 203-12.

127. ORTLEPP (R. J.). - Bigalkenema namaquensis, Gen and Sp. Nov., a Trichotrongylld worm from sheep. Onderstepoort J. vet. Res., 1963, 30 (1) : 119-24, 1 fig.

128. O'ROKE (E. C.). - The relation of parasitism to wild life Conservation. J. Porasit., 1927, $14: 135$.

129. OTTO (R.) . - Beitráge zur anatomie und histologie der Amphistomeen. Gastrothylax gregarius Looss, Gastrothylax crumenifer Creplin, Amphistomum conicum Rudolphi, Amphistomum bothriophoron Braun, Amphistomum gigantocotyle Brandes, Amphistomum subtriquetum Rudolphi, Gastodiscus polymastos Leuckarl. Inaug. Dissert. Philosoph. Facultät Univ. Leipzig. Dt. Zeitschr. f. Thiermedicin u. vergleıch. Pathologie., 1896, $22: 1-78,30 \mathrm{fig}$.

130. PELLEGRINI (D.) (a). - El Cyst. dromedarius nel bovino. Racc. Stud. Vet. Path. Somoli, 1942 (1) : 1-2.

131. PELLEGRINI (D.) (b). - Cysticercosi del camello. Rocc. Stud. Vet. path. Somali, 1942 (1) : 42-8.

132. PELLEGRINI (D.) (o). - II dromedarii $n$. sp. nel camello e relativa Cysticercosis. Boll. Soc. 1t. Med. Ig. Trop. Asmara, 1947, 7 (3-4) : 317-24.

133. PELLEGRINI (D.) (b). - II C. dromedarii Pellegrini 1945 nel bovino. Boll. Soc. It. Med. Ig. Trop. Asmoro, 1947, 7 (5-6) : 550-3.

134. PELLEGRINI (D.) (c). - II dromedarii Pellegrini 1945 e lo stato larvale della Taenia hyaenae, Baer 1927. Boll. Soc. It. Med. Ig. Trop. Asmoro, 1947, 7 (5-6) : 554-65.

135. PELLEGRINI (D.) (d). - Nel bovino la sede di predilezione del C. dromedarii Pellegrini 1945 e nei gangli mesenterici. Boll. Soc. It. Med. Ig. Trop. Asmara, 1947, 7 (5-6) : 566-72.
135'. PELLEGRINI (D.). Le Cysticercus dromedarii du chameau et des bovins et le Taenia bryanca correspondant de l'hyène (BAER, 1927). Bull. off. Int. Epiz., 1950, 33. (1-2), 21-27.

136. PESTER (F. R. N.). - Coopericides sp. inq. producing nodules in the small intestine of a Thomson's gazelle. Trons. R. Soc. Trop. Med. Hyg., 1926, 65 (4) : 267.

137. PRUDHOE (S.). - Exploration du Parc de I'Upemba - Mission G. F. de Witte Trématodes. Inst. Porcs Nat. Congo Belge, 1957, 48, 27 p., 7 fig.

138. RAILLIET (A.). - Sur quelques Sclérostomiens parasites des ruminants ef des porcs. C. R. Soc. Biol., 1902, 54 : 107.

139. RAILLIET (A.) et HENRY (A.). - Sur une Filaire péritonéale des porcins. Bull. Soc. Paih. Exot., 1911, 4, 486.

140. RAMANUJACHARI (G.), ALWAR (V.S.). Bunostomem bhavanagarensis $n$. $\mathrm{sp}$. Ind. vet. J., 1950, 27 (4) : 241-43, 6 fig., 1 tabl.

141. RODE (P.). - Faune de l'empire français. II : Les Mammifères Ongulés de l'Afrique Noire. Paris, 1943, 121 p., 91 fig.

142. RODENWALDT (T.). - Filaria kuelzii n.sp. Arch. Schiffs. U. Trop. Hyg., 1910, 24 : 52935, 6 fig.

143. RODHAIN (J.) et GILLIAN (J.). - Présence de nodules à Onchocerques chez un buffle du Cap dans le Haut Ituri. Ann. Soc. Méd. Trop., 1938, $18:$ 85-88.

144. RODHAIN (J.). - Un deuxième cas d'Onchocercose nodulaire chez le buffle du Cap. Syncerus caffer dans le Haut-lturi. Ann. Soc. belge. Méd. Trop., 1944, 24 : 43-53.

145. ROUND (M. C.). - The Helminth parasites of domesticated animals in Kenya. J. Helminth, 1962, 36 (4) : 375-449.

146. SAMBON (L.). - A synopsis of the c. family Linguatulidae. J. Trop. Med. Hyg., 1922, 391-428.

147. SANDGROUND (J. H.). - Notes and descriptions of some parasitic Helıninths collected by the expedition. Rep. Harward Exp. Afr. Rep. Liberia a. Congo Belge, 1929, 46281, et 397-99. 
148. SANDGROUND (J. H.). - A note on Phacochoerostrongylus pricei Schwartz 1928 and on the male of Fsophagostomum goodeyi, Daubney 1926. Ann. Trop. Med. Porosit., 1937, $31: 23-4$.

149. SARWAR (M. M.). - A criłical survey of the representation of the genus Trichuris in ruminanis in Indo-Pakistan. Acto Trop., 1957, 14 (3) : 225-7.

150. SARWAR (M. M.). - Reconstruction of the genus Trichuris and a short review of its taxonomy and morphology. Biologica, 1959, $5: 19-35$.

151. SCHULZ $(H$.$) . - Sur la faune helmintho-$ logique de Gazella subgutturosa. Ann. Parasit. hum. comp., 1928, 6:101-4.

152. SING (P. P.), PANDE (B. P.). - Helminths collected from the Indian Antelope, Antelope cervicapra. Ann. Parasit. hum. comp., 1963, 38 (3) : 439-57, 18 fig.

153. SKRJABIN (K. l.) et ORLOV (I. V.). - Trichostrongylidae of ruminants. Moscou, 1934, $351 \mathrm{p}$.

154. SKRJABIN (K. I.) ef SHIKHOBALOVA ( $N$. $P$.$) . - A new rearrangement of the Taxo-$ nomy of the Nematodes belonging to the family Filariidae. C. R. Acod. Sci. U.R.S.S., 1945, $49: 690-2$.

155. SKRJABIN (K. I.). - Trématodes des animaux sauvages ef domestiques (en Russe). Akad. Nouk. C. C. C. P., 1949, 3 : 624 p., 145 fig. 4 pl.

156. SKRJABIN (K. t.), SHIKHOBALOVA (N. P.) et Coll. - Classification key of Parasitical Nematodes. Vol III. Strongylidés. U.R.S.S. Acad. Sci., Moscou, 1952, 890 p.

157. SKRJABIN (K. I.) et SHIKHOBALOVA (N. P.) et SHUL'TS (R. S.) 1954. - Essentials of Nematodology. III. Trichostrongylidés of animals and man. Acad. Sci. U.R. S. S. Moscou (Isroel Prog. Sci. Transl. 1960), 693 P., 386 fig.

158. SOLOMON (G.). - On a collection of parasitic worms from East Africa. J. Helminth., 1932, 10 (4) : 209-30.

159. SMIT (H.J.) et NOTOSOFDIRI (R.), - Einige Strongyliden onzer huis dieren. Nederl. Bl. e. Diergeneesk., 1923, 25 (2-3) : 191-8.
160. SOUTHWELL (T.). - Notes on the anatomy of Stılesia hepatica and on the genera of Subfamily Thysanosominae (including Avitellinae). Ann. Trop. Med. Parasit., 1929, 23 : 47-66.

161. SOUTHWELL (T.). - The Fauna of British India. Vol II Cestoda. London, 1930, 262 p., 350 fig.

162. SPASSKY (A. A.) 1961. - Anoplocaphalata Cestodes of Domestic and wild animals. Principles of Cestodology (Moscou 1954). 1 : 783 (Jerusaiem : Israel program Sci. transl.).

163. SPENA (A.). - Sopra un Cestode parassita della Gazella. Nuov. Vet., 1935, $13: 21-4$.

164. SPREHN (C. E. W.). - Lerhbuch der Helminthologie. Berlin, 1932, $998 \mathrm{p}$.

165. STILES (C. W.), GOLBERGER (J.). - A Study of the anatomy of Watsonius $(\mathrm{Ng})$ watsoni of man and of nineteen allied species of mammalian Trematodes worms of the Superfamily Paramphistomoidea. Treas. Dept. Public. Health. Mar. Hosp. Serv. U. S. Hyg. Lab., 1910 (60) : 1-264, 205 fig.

166. STRONG (R. P.) SHATTUCK (G. C.), Animal infections. Afr. Rep. Liberia a. Belg. Congo, Dep. Trop. Med: Inst. Trop. Biol. Med,, 1930, 5 (1) : 412-61.

167. STUNKARD (H. W.). - The parasitic worms collected by the American museum of natural hustory expedition to the Belgian Congo. 1909-14 Part I Trematoda. Bull. Amer. Mus. Nat. Hist., 1929, 58 (6) : 233-89, $37 \mathrm{fig}$.

168. SZIDAT (L.). - Parasiten aus Liberia und französisch-Guinea. II-Teil-Trematoden. Z. Parasitenk, 1932, 4 (3) : 506-11.

169. STILES (C. W.), HASSALL (A.), - A revision of the adult Cestodes of cattle, sheep and allied animals. U. S. Dep. Agri. Bull., 1893 (4) : 73-9.

170. TAYLOR (D. C.). - Cysticercosis in an Oryx. Vet. Rec., 1958, 70 (51) : 1207.

171. TENDEIRO (J.). - Subsidios paro o conhecimento da Fauna parasitologica da Guiné. Bol. Cult. Guné, 1948, 3 :,638-78.

172. TENDEIRO (J.), - Adualidade veterinaria da Guiné portugesa. Bissau, 1951, 213 p. 
173. THEILER (G.). - On the classification of the Cestode genus Moniezia, Blanchard 1891. Ann. Trop. Med. Porosit., 1924, 18 : 109-23, 12 fig.

174. THORNTON $\left(H_{\text {. }}\right)$. - A review of the Oesophagostome in the collection of the Liverpool school of Tropical medicine. Ann. Trop. Med. Porasit., 1924, 18 : 393-407.

175. TRAVASSOS (L.). - Synopse des Paramphistomoidea. Memor. Inst. Oswal. Cruz., 1934, 29 (1) : 19-178, 86 fig.

176. TRAVASSOS (L.). - Revisao da Familia Trichostrongylidae Leiper 1912. Monogr. Inst. Oswaldo Cruz., 1937 (1) : 512.

177. TRAVASSOS (L.). - Revisao da familia Dicrocoelidae Odhner 1901. Monogr. Inst. Oswaldo Cruz., 1944 (2) : 357.

178. TWAITE (J. W.). - The genus setaria. Ann. Trop. Med. Parasit., 1927, 21: 427-66, 20 fig.

179. URQUARTH (G. M.), ZAPHIRO (D. R. P.), SPINAGE (C. A.). - Some internals parasites of game animals in Kenya. East. Afr. Agri. For. Ja., 1960, 26 (1) : 11-20, 4 pl.

180. VAN DEN BERGHE (L.) ef VUYLSTEKE (C.). - Quelques setaires du Congo Belge avec la description d'une espèce nouvelle du potamochère. Rev. Zool. Bot. Afr., 1936, 28 (4) : 421-30.

181. VAN DEN BERGHE (L.). - Shistosoma bovis chez deux Antilopes, Limnotragus spekei (parc national de la Kagera Ruanda sous mandat Belge). Ann. Parasit. hum. comp., 1937, 15 (6) : 518-19.

182. VAN DEN BERGHE (L.). - Les Shistosomes et les Shistosomoses au Congo Belge et dans les territoires du Ruanda-Urundi. Inst. Roy. Col. Belg., 1939, 8 (3) : 153, 27 pl.

183. VAN DEN BERGHE (L.). - Exploration du pare national Albert et du parc national de la Kagera, Mission Van den Berghe (1936). II: Helminthes parasites. Inst. Parcs Nat. Congo Belge, Bruxelles, 1943 (2) : 1-30, $11 \mathrm{pl}$.

184. VEVERS (G. M.) 1922. - On the parasitic Nematodes collected from mammalian hosts which died in the gardens of the zoological society of London during the year 1919-1921.
185. VUYLSTEKE (C.). - Notes sur quelques Nématodes parasites avec description de neuf espèces nouvelles. Rev. Zool. Bot. Afr., 1956, 53 (3-4) : 441-73, 87 fig.

186. WARDLE (R. A.), MC LEOD (J. A.), The zoology of Tapeworms. University of Minnesota, 1952, 780 p., 419 fig.

187. WARE (F.). - Two uncommon Nematodo parasites of cattle. J. Comp. Poth. Therap., 1925, 38 (2) : 83-9.

188. WOLFFHUGEL (K.). - Stilesia hepatica $N$. $S p$. ein bandwurm aus den gallengàngen von schâfen und ziegen Ostafrikas. Berlın Tierärzt. Wochenschr., 1903, 43 : 1-16.

189. WOODLAND (W. N. F.). - On three new species of Avifellina (Cestoda) from India and the Anglo-Egyptian Sudan, with a redescription of the type species $A$. centripunctata (Rivolta, 1874). Ann. Trop. Med, Parasit., 1927. 21 : 385-414.

190. WOODLAND (W. N. F.). - On a new genus of Avitellina Tapeworm from ruminants of East Africa. Parasitology, 1928, 20 : 56-65, 20 fig., $2 \mathrm{pl}$

191. WOODLAND (W. N. F.). - A new species of Avitellina Tapeworm, Avitellina sandgroundi from Hippotragus equinus. Ann. Trop. Med. Porasit., 1935, 29 : 185-90.

192. YAMAGUTI (S.). - Studies on the Helminth fauna of Japan. Part. 27. Trematodes of mammals. Jap. J. med. ScI., VI Bact. and Parasitol., 1939, 1 (3) : 131-51, 12 fig.

193. YAMAGUTI (S.) (1958). - Systema Helminthum, Vol. I. The digenetic Trematodes of Vertebrates. New York et London, Interscience publishers, 1958, 979 p. Part. IPart. II. pp. 980-1573.

194. YAMAGUTI (S.). - Systema Helminthum. Vol. II. The cestodes of Vertebrates. New York and London-Interscience publishers, Inc. 1959, $860 \mathrm{p}$.

195. YAMAGUTI (S.). - Systema Helminthum. Vol. III. The Nematodes of vertebrates. New York, interscience Publishers, Inc., 1961, Part. I, 679 p. ; Part. II, pp. 681-1261. 
196. YEH (L. S.) (a). - A new filariid Gazellofilaria tanganyikae Gen and Sp. Nov. with cuticules bosses. Trans. R. Soc. Trop. Med. Hyg. 1955, $49: 296$.

197. YEH (L. S.) (b). - On a collection of Helminths from Thomson's Gazelle, Gazella thomsoni from Tanganyika. J. Helminth., 1955, 29 (4) : 203-28, 45 fig.

198. YEH (L. S.) (1958), - - On the identity of the Filarial worms Setaria hornbyi Boulenger 1921 and Setaria twatei, Mönnig, 1931. Trons. Roy. Soc. Trop. Med. Hyg., 1958, 52 (4) : 297.

199. YEH (L. S.) - - A revision of the Nematode genus Setaria Viborg 1795 - lts hostparasite relatioship, speciation and evolution. J. Helminth., 1959, 53 (1): 1-98, 185 fig.

200. YORKE (W.), MAPLESTONE (P. A.). - The Nematodes parasites of Vertebrates. London, 1926, 536 p., 307 fig.

\section{Addendum}

Deux intéressants mémorres sont parvenus trop tard pour être incorporés dans le texte. II s'agit de :

1. LE VAN HOA. - Nématodes parasites des Mammifères, Reptiles et Amphibiens du Congo. Phasmidiens. Exploration du pare national de I'Upemba. Mission $G$ de WITTE (1946-49). Bruxelles, 1962 (65), 58 p.

2. GRÉTILLAT (S.). - Sur quelques Paramphistomatoided (Trematoda) d'une collection du Musée royal de l'Afrique centrale. Rev. Zool. Bot. Afr., 1964, 69 (3-4) : 351-57, 8 fig.

Les auteurs étudient un certain nombre de Nématodes et de Trématodes d'Artiodactyles sauvages de la République démocratique du Congo recueill is dans le parc national de l'Upemba et dans I'Uélé. 